\title{
Berufswechsel beim \\ Übergang von der Lehre in den Arbeitsmarkt
}

\section{Barbara Müller und Jürg Schweri}

Anhand des Jugend-Längsschnittdatensatzes TREE auf der Basis der PISA-2000-Erhebung untersuchen wir das Vorkommen, die Gründe und Auswirkungen von Berufswechseln im Übergang von der Lehre in den Arbeitsmarkt. Rund 9 Prozent der Lehrabsolventen, welche ein Jahr nach Lehrabschluss erwerbstätig sind, arbeiten in einem wesentlich anderen Beruf als dem erlernten. Als wichtigste Einflussfaktoren auf diese Berufswechsel erweisen sich die Charakteristika des erlernten Berufs, sowie wie Anforderungsniveau und Arbeitslosigkeit im Beruf und die Zufriedenheit mit der Ausbildung. Wenig Einfluss haben der persönliche Hintergrund und die Fähigkeiten der Absolventlinnen, gemessen an den Lehrabschlussnoten und den PISATestscores.

Die Berufswechslerlinnen verdienen im Schnitt rund 5 Prozent weniger als jene, die auf dem erlernten Beruf arbeiten. Dies weist darauf hin, dass in der beruflichen Grundbildung eine Spezialisierung stattfindet; das erworbene berufsspezifische Humankapital wird bei einer Tätigkeit im erlernten Beruf vom Arbeitsmarkt vergütet. Allerdings sind die Lohnwirkungen von Berufswechseln nicht einheitlich. Im Schnitt verdienen Berufswechslerlinnen zudem signifikant mehr als Ungelernte. Vergleicht man dual und vollschulisch ausgebildete Personen, zeigt sich bei letzteren ein generell leicht tieferer Lohn sowie eine höhere Wahrscheinlichkeit, den Beruf zu wechseln. Nach Berufswechseln zeigen sich für die beiden Gruppen im Schnitt keine Lohnunterschiede mehr.

\section{Einleitung ${ }^{1}$}

Berufsbildungssysteme gelten im Vergleich zu anderen Ausbildungsformen als sehr erfolgreich bei der Integration von Jugendlichen in den Arbeitsmarkt, insbesondere aufgrund der vergleichsweise tiefen Jugendarbeitslosigkeit in Ländern mit ausgebauter Berufsbildung (OECD, 2000; Ryan, 2001). Das liegt einerseits an der betrieblichen Sozialisierung der Lernenden, andererseits an der beruflichen Spezialisierung; beide Elemente machen die Lehrabsolventen aus Arbeit- 
gebersicht zu interessanten Fachkräften. Diesen Vorteilen beim Eintritt ins Erwerbsleben stehen jedoch Befürchtungen gegenüber, dass die Anpassungsfähigkeit an wirtschaftsstrukturelle und technologische Veränderungen bei dual Ausgebildeten eingeschränkt sein und die früh stattfindende berufliche Spezialisierung längerfristig ein Mobilitätshemmnis auf dem Arbeitsmarkt darstellen könnte (Winkelmann, 2006). Flexibilität und eine breite Einsetzbarkeit der erworbenen Kompetenzen können allerdings bereits beim Übergang in den Arbeitsmarkt gefragt sein: das betriebliche Ausbildungskalkül orientiert sich nicht notwendigerweise am künftigen Fachkräftebedarf, so dass die Zahl der Ausgebildeten die Nachfrage nach Berufsleuten in bestimmten Berufen übersteigen kann (Stichwort «Ausbildung über Bedarf»).

Der beruflichen Mobilität der Lehrabsolvent-/innen kommt vor diesem Hintergrund eine wichtige Bedeutung zu. Auswertungen der Schweizer Volkszählungsdaten 1970 bis 2000 zeigen, dass Berufswechsel von Personen mit Berufslehre zugenommen haben und am ehesten im Alter zwischen 21 und 22 Jahren stattfinden (Sheldon, 2005). Dies kann als Anpassungsprozess an den berufsstrukturellen Wandel interpretiert werden, der nicht nur am ersten Übergang (von der Schule in die Berufslehre) stattfindet, sondern auch durch Berufswechsel nach der Lehre beim Übergang in den Arbeitsmarkt (vgl. dazu auch Leemann und Keck, 2005). Für Veränderungen in Auf- und Abstiegsrisiken verschiedener Berufe im Laufe der Zeit vgl. Buchmann (1996).

Der «zweite» Übergang, d.h. der Übergang von der beruflichen Grundbildung in den Arbeitsmarkt, stellt somit nicht nur hinsichtlich des Arbeitslosigkeitsrisikos eine kritische Phase dar, sondern auch in Bezug auf die Frage, wie gut die Lehrabsolventen das Erlernte auf dem Arbeitsmarkt einsetzen können. Zur Beurteilung der Funktionsfähigkeit des dualen Ausbildungssystems ist es wichtig zu wissen, welche Personen warum und mit welchen Konsequenzen ihren erlernten Beruf verlassen. Dazu ist bislang für die Schweiz wenig bekannt. Die vorliegende Arbeit soll anhand des Längsschnittdatensatzes TREE ${ }^{2}$ mit der Betrachtung von Lehrabgängerinnen und Lehrabgängern rund ein Jahr nach Lehrabschluss dazu beitragen, Einblick in die Berufswechselvorgänge an der zweiten Schwelle zu erhalten.

Weder aus individueller noch aus gesellschaftlicher Sicht ist dabei a priori feststellbar, ob ein Wechsel nach der Lehre für die Betroffenen als nützlich oder schädlich zu betrachten ist. ${ }^{3}$ Verschiedene Arbeitsmarkttheorien zeigen etwa, dass die Wechselgründe sehr vielfältig sein können und sowohl positive wie negative Lohnfolgen von Berufswechseln möglich sind. Die Analysen in diesem Beitrag widmen sich daher sowohl den Determinanten von Berufswechseln nach der Lehre wie auch den Lohnfolgen dieser Wechsel. Duale Lernende werden dabei mit Lernenden aus vollzeitschulischen beruflichen Ausbildungen verglichen, da diese Ausbildungen auch eine bildungspolitische Alternative zur dualen Lehre darstellen. ${ }^{4}$

Gleichzeitig muss berücksichtigt werden, dass Lehrabsolventen nicht nur die Wahl zwischen einer Stelle auf dem erlernten Beruf und einem Berufswechsel 
haben, sondern auch Alternativen wie der weitere Verbleib im Bildungssystem oder Arbeitslosigkeit in Frage kommen. Diesem Umstand wird in der vorliegenden Studie durch die breite Abbildung der möglichen Alternativen und ihrer Determinanten Rechnung getragen. Die reichhaltigen Angaben im TREE-Datensatz erlauben es dabei, die Heterogenität der Personen in den Schätzungen abzubilden. Für die rund 1800 Lehrabgänger/innen liegen nebst Informationen zu ihrer Tätigkeit nach der Lehre und zum Lehrberuf auch Informationen zur persönlichen Einschätzung von Ausbildungszufriedenheit und Berufsverbundenheit, zu persönlichen Merkmalen und zum sozio-ökonomischem Hintergrund sowie zu Leistungsindikatoren (PISA-Testscores, Lehrabschlussnote) vor.

Der Beitrag gliedert sich wie folgt: Nach einer Diskussion wichtiger arbeitsmarktökonomischer Theorien, die für die Analyse von Berufswechseln relevant sind, wird die hauptsächlich für Deutschland vorhandene empirische Literatur zu Berufswechseln im Zusammenhang mit dem Berufsbildungssystem dargestellt. Daraus werden für die vorliegende Analyse zentrale Arbeitshypothesen abgeleitet. Nach einer Beschreibung der Datengrundlage und der Schätzstrategie werden die Ergebnisse der Analysen einerseits zu den Gründen von Berufswechseln, andererseits zu ihren Auswirkungen vorgestellt. Abschliessend werden die Resultate zusammengefasst und ihre bildungspolitischen Implikationen hinsichtlich der dualen Grundbildung diskutiert.

\section{Arbeitsmarktökonomische Theorien zur Analyse von Berufswechseln}

Innerhalb der ökonomischen Bildungsliteratur dominiert der Analyserahmen der Humankapitaltheorie von Becker (1962). Die Ausbildung wird als Investition der Lernenden in ihr Humankapital begriffen. Dank der produktivitätssteigernden Wirkung von Bildung steht der getätigten Investition (insbesondere Lohnverzicht während der Ausbildung) ein höherer Lohn nach der Ausbildung gegenüber. Die von Becker eingeführte theoretische Unterscheidung des akkumulierten Humankapitals nach seinem Spezifitätsgrad - firmenspezifisch versus allgemein verwertbares Humankapital - hat wichtige Implikationen für die Mobilität der Arbeitnehmer/innen: der spezifische Anteil des Wissens kann per Definition nicht über den Stammbetrieb hinaus transferiert werden und büsst seinen Wert in anderen Firmen vollständig ein. Er ist somit ein Mobilitätshemmnis. Allgemeines Humankapital hingegen wird auf dem ganzen Arbeitsmarkt gleichermassen entlöhnt.

In der jüngeren Literatur wird der Spezifitätsbegriff5 auf berufs- oder branchenspezifisches Humankapital ausgedehnt (Dustmann und Meghir, 2005; Kambourov und Manovskii, 2008; Neal, 1995; Parent, 2000; Shaw, 1987). Wenn die in der Berufslehre erworbenen Kenntnisse und Fähigkeiten eine berufsspezifische Komponente aufweisen, kann dieser Teil des Humankapitals in 
einem anderen Beruf nicht eingesetzt werden. Berufswechsel sind folglich mit Lohneinbussen verbunden. Freiwillige Berufswechsel sind innerhalb dieses bewusst sparsam formulierten Theorierahmens nicht zu erklären; die Lohnveränderung beim Berufswechsel gibt jedoch Aufschluss darüber, wie transferierbar das durch das Berufsbildungssystem vermittelte Humankapital ist, beziehungsweise wie hoch die Prämie für berufsspezifisches Humankapital ist.

In US-amerikanischen Studien hat sich gezeigt, dass das Ausprobieren mehrerer Stellen zu Beginn des Erwerbslebens («job shopping») eine bedeutende Quelle für individuelle Karriere- und Lohnaufstiege darstellt (Topel und Ward, 1992). Rubinstein und Weiss (2007) betonen in einem Überblicksartikel, dass Wechsel vor allem für schlechter Ausgebildete oft zu Stellen in besser bezahlten Berufen und Branchen münden. Search- und Matchingmodellen zufolge können solche freiwilligen Betriebs- und Berufswechsel als Ergebnis eines Suchprozesses angesehen werden, der darauf abzielt, die eigenen Fähigkeiten auf dem Markt möglichst optimal und gewinnbringend einzusetzen (Jovanovic, 1979; Mortensen, 1986; Neal, 1999). Innerhalb dieses Analyserahmens wird ein Betriebs- oder Berufswechsel dann vollzogen, wenn die stellensuchende Person in einem anderen Betrieb oder Beruf ein Stellenangebot erhält, das besser ist als das beste Angebot im Stammbetrieb bzw. im erlernten Beruf. Die Lohndifferenz ${ }^{6}$ zwischen dem Status quo und der neuen Stelle ist zugleich Grund wie auch Ergebnis des Wechsels. Von allen potenziellen Wechselmöglichkeiten werden jene realisiert, die dem Individuum einen höheren Lohn versprechen. Daraus folgt, dass ein positiver Zusammenhang zwischen realisierten Berufswechseln und Löhnen zu erwarten ist, was dem oben beschriebenen, negativen Humankapitaleffekt entgegen läuft und diesen überlagern kann.

\section{Literatur zu Berufswechseln nach einer Berufs- ausbildung}

Es gibt eine wachsende internationale Literatur zum Übergang von der Ausbildung in das Erwerbsleben (Bertschy, Cattaneo und Wolter, 2008; Bradley und Nguyen, 2004; Ryan, 2001 und die dort zitierte Literatur). Mit Bezug auf die Berufsausbildung von besonderem Interesse sind empirische Studien, die sich dem Vergleich von dual ausgebildeten Lernenden mit Absolventen von Vollzeitberufsschulen widmen. Einige Studien finden geringere Transitionsprobleme für Absolvierende dualer Lehren (z.B. Bertschy et al., 2008, für die Schweiz; Bonnal, Mendes und Sofer, 2002, für Frankreich; Büchel, 2002; Franz, Inkmann, Phohlmeier und Zimmermann, 2000; Winkelmann, 1996, für Deutschland), allerdings ist die empirische Evidenz zu Vorteilen des dualen Berufsbildungssystems nicht eindeutig (Ryan, 1998; 2001). Plug und Groot (1998) finden für die Niederlande in der langen Frist keine Unterschiede zwischen dualen und schulischen Berufsausbildungen in gleichen Berufen, weder bezüglich Beschäftigungs- 
chancen, Löhnen noch Lohnentwicklungen. Die niederländischen Arbeitgeber schätzen in Befragungen die Qualität der dual Ausgebildeten eher höher ein (Smits und Zwick, 2004). Hartog, Jonker und van Ophem (2002) finden aber für den Fall der holländischen Wirtschaftsprüfer mehr Mobilität und steilere Lohnprofile für Absolventen vollzeitschulischer Bildung gegenüber den dual Ausgebildeten. In einer deutsch-schwedischen Vergleichsstudie von Korpi und Mertens (2003) zeigt sich eine höhere berufliche Mobilität für Beschäftigte mit vollzeitschulischer Berufsausbildung.

Für Deutschland, dessen Berufsbildungssystem viele Parallelen mit dem schweizerischen aufweist, liegen einige Studien zum Thema Berufswechsel vor, die grossteils auf den Daten der BIBB-IAB-Befragung basieren. Sie beziehen sich nicht explizit auf Berufswechsel am Übergang in den Arbeitsmarkt, sondern auf Berufswechsel über die gesamte Erwerbskarriere. Büchel und Neubäumer (2001) finden, dass knapp ein Viertel der Lehrabsolventen zwischen ein bis zwölf Jahren nach Lehrabschluss den Beruf gewechselt hat. Als Grund für den Wechsel gaben 10\% der Befragten den Mangel an Stellen im Ausbildungsberuf an, von den übrigen Personen wurden andere Gründe genannt, z.B. die Möglichkeit ein höheres Einkommen zu erzielen (25\%), veränderte Interessen (29\%) oder mehr Einfluss und Verantwortung (8\%). Eine Mehrheit von 59\% bewertet den Wechsel als deutliche Verbesserung der beruflichen Situation, nur 8\% sprechen von einer Verschlechterung.

Dustmann und Schönberg (2007) werten die Frage nach der Anwendbarkeit der in der Berufslehre erworbenen Fertigkeiten in der aktuellen Arbeitstelle aus und finden, dass diese von Berufswechslern rund 35\% tiefer eingeschätzt wird. Nebst diesem bedeutenden berufsspezifischen Anteil ist die betriebsspezifische Komponente von 5\% klein; Lohnfolgen werden nicht analysiert. Werwatz (2002) findet unter Berücksichtigung von Selektionseffekten, dass nur für eine Minderheit der Befragten ein Berufswechsel mit negativen Lohnkonsequenzen verbunden ist, nämlich wenn sie nur wenige der erlernten Fertigkeiten im neuen Beruf anwenden können. Die Mehrheit der Wechsler arbeitet aber auf einer qualifizierten Stelle mit Lohngewinnen gegenüber Nicht-Wechslern. Auch Fitzenberger und Spitz (2004) finden, dass Berufswechsel mehrheitlich vollzogen werden, um grössere Verdienstchancen wahrnehmen zu können. So hängen Berufswechsel negativ vom Durchschnittslohn im Ausbildungsberuf und positiv vom Durchschnittslohn im Erwerbsberuf ab. Wird dieser Zusammenhang mit Hilfe eines Selektionsmodells berücksichtigt, dann ist der (kausale) Lohneffekt eines Berufswechsels im Schnitt immer noch positiv, jedoch nicht signifikant, weil stark altersabhängig: in jungen Jahren sind Berufswechsel eher mit negativen Lohnkonsequenzen verbunden, mit zunehmendem Alter erweisen sich Wechsler als positive Selektion in Bezug auf die Löhne. Weiter kommen Clark und Fahr (2002) anhand des Sozio-ökonomischen Panels in ihrer Analyse «unfreiwilliger» Berufswechsler (aus Betriebsschliessungen) zum Schluss, dass das vermittelte Humankapital einer Berufslehre innerhalb eines breit definierten Be- 
rufsfeldes vollständig transferierbar ist und dass erst Wechsel zwischen weit entfernten Berufen (Wechsel auf der 1-Steller-Ebene innerhalb der deutschen Klassifizierung der Berufe) in Lohneinbussen von bis zu einem Drittel des Wertes einer Berufslehre resultieren.

\section{Hypothesen über Gründe für und Lohnfolgen von Berufswechseln an der 2. Schwelle}

Aus den oben dargestellten Such- und Matchingmodellen ergibt sich eine erste Kategorie von Berufswechselgründen, nämlich solche, welche auf eine Verbesserung der individuellen beruflichen Situation abzielen. Mehr Berufswechsler sollten demnach dort zu erwarten sein, wo berufliche Verbesserungen am ehesten möglich sind: Einerseits, wenn der «Match» einer Person mit dem Lehrberuf schlecht war (tiefere Zufriedenheit und Verbundenheit mit dem Beruf), andererseits, wenn der zu erwartende Lohn im Lehrberuf niedrig ist.

Die zweite Kategorie von Berufswechselgründen bezieht sich auf Merkmale der Ausbildungsberufe. Diese lassen sich teilweise mit Hilfe der Humankapitaltheorie charakterisieren: Die Humankapitaltheorie liefert zwar wie erwähnt keine direkten Begründungen für Berufswechsel, zeigt aber auf, dass sich Lehren im Anteil von generellem und spezifischem Humankapital unterscheiden, was zu unterschiedlichen betrieblichen Ausbildungsstrategien und -zielen führt. Bei Lehren, bei denen die Betriebe während der Lehrzeit Nettokosten tragen, steht das Ziel im Vordergrund, mindestens einen Teil der Lernenden nach Lehrabschluss zu übernehmen, um Nutzen durch Übernahmen zu generieren (Wolter und Schweri, 2004) und so die investierten Kosten zu decken. Entsprechend sind bei diesen Lehren erheblich weniger Betriebs- und Berufswechsel zu erwarten als bei Lehren, wo die Nettokosten dank der produktiven Arbeit der Lernenden bereits bei Lehrabschluss gedeckt sind und keine Notwendigkeit zur Übernahme durch den Lehrbetrieb besteht. Da Lehrabsolventen aus kleinen Lehrbetrieben seltener im ehemaligen Lehrbetrieb weiterbeschäftigt werden können, lässt sich weiter vermuten, dass sie ebenfalls eher Mühe bekunden, eine Anstellung auf dem Beruf zu finden.

Ein wichtiges Merkmal der Ausbildungsberufe ist auch die berufsspezifische Arbeitslosigkeitsrate, welche die Beschäftigungsaussichten der Lehrabsolventen und -absolventinnen beim Übergang in den Arbeitsmarkt bestimmt. Die Arbeitslosigkeit im Beruf wird, abgesehen von der gesamtwirtschaftlichen Arbeitslosenrate, unter anderem von wirtschaftlichen Schocks (z.B. technologischer Wandel, Globalisierung) beeinflusst. Auch die betrieblichen Ausbildungsentscheidungen sind jedoch nicht zwangsläufig auf den Fachkräftebedarf am Arbeitsmarkt ausgerichtet. Bei Berufen mit hohen Nettokosten werden gemäss einer Erweiterung der Humankapitaltheorie (Acemoglu und Pischke, 1999) weniger Lernende ausgebildet als gesamtwirtschaftlich optimal wäre. Umgekehrt kann es in produktionsorientierten Berufen zu einer "Ausbildung über Bedarf» kommen. 
Die dritte Gruppe der Berufswechselgründe umfasst individuelle Charakteristika der Lernenden. In den Schätzungen werden die sozioökonomische Herkunft, das Geschlecht, der Migrationshintergrund und weitere individuelle Merkmale berücksichtigt. Speziell zu erwähnen sind jene Variablen, welche die Fähigkeiten («ability») der Jugendlichen erfassen, da Betriebswechsler gemäss einer vielzitierten Studie für Deutschland eine Negativauswahl unter den Lehrabsolventen darstellen (Acemoglu und Pischke, 1998). Dank den Variablen Literacy-Testscore aus PISA-2000 und der Lehrabschlussnote lässt sich für die individuellen Fähigkeitsunterschiede kontrollieren und die Hypothese testen, ob es sich bei Berufswechsler/innen um eine negativ selektierte Gruppe handelt.

Welcher durchschnittliche Lohneffekt für die beobachteten Berufswechsel erwartet wird, ist theoretisch nicht eindeutig und hängt davon ab, ob der negative Effekt des nicht in einen anderen Beruf transferierbaren berufsspezifischen $\mathrm{Hu}-$ mankapitals oder der positive Effekt freiwilliger, nutzbringender Wechsel überwiegt, wie sie von Such- und Matchingmodellen postuliert werden. Der erstmalige Arbeitsmarkteintritt stellt aufgrund der geringen Arbeitsmarkterfahrung der Jugendlichen und der Gefahr eines Stigmatisierungseffekts bei früher, länger andauernder Arbeitslosigkeit eine kritische Phase dar. Während die Jugendlichen daher auf eine rasche, erfolgreiche Stellensuche angewiesen sind, sind die Stellenangebote in manchen Berufen eher rar. Es ist daher davon auszugehen, dass ein nicht zu vernachlässigender Teil der Lehrabgänger und -abgängerinnen in dieser Übergangssituation den Beruf mangels Alternativen wechselt und dabei eine Lohneinbusse in Kauf nehmen muss. Die Höhe der Einbusse hängt dabei gemäss Theorie davon ab, wie gut das in der Ausbildung erworbene Humankapital im neuen Beruf eingesetzt werden kann.

Für Absolventen vollzeitschulischer Berufsbildungen vermuten wir einen höheren Anteil an allgemeinbildendem und somit transferierbarem Wissen. Dies sollte sich in einer höheren beruflichen Mobilität niederschlagen, welche im Schnitt mit geringeren Lohnnachteilen als bei Absolventen dualer Berufsbildungen verbunden ist.

\section{Daten und Operationalisierung}

Empirische Grundlage für die vorliegende Analyse bildet der Datensatz TREE (TRansitionen von der Erstausbildung ins Erwerbsleben). TREE ist auf nationaler Ebene die erste und bislang einzige Erhebung mit Längsschnittcharakter, welche die nachobligatorischen Ausbildungsverläufe junger Menschen und deren Einstieg ins Erwerbsleben erfasst. Diese Daten umfassen jährlich wiederkehrende Nachbefragungen der Jugendlichen, die im Jahr 2000 an der ersten Schweizer PISA-Befragung (OECD, 2002; BFS, 2002) teilnahmen und im selben Jahr ihre obligatorische Schulzeit beendeten ${ }^{7}$. Nebst detaillierten Angaben 
zu Bildungs- und Erwerbsverläufen in den Jahren nach Abschluss der obligatorischen Schule liegen somit zu den Befragten auch PISA-Informationen wie der «Literacy»-Testscore und ein reichhaltiges Set von individuellen Hintergrundsvariablen vor. Nutzbar für die vorliegende Analyse sind die ersten fünf Befragungswellen von TREE. Dies erlaubt, den Grossteil der Personen in der PISA2000 Stichprobe zu analysieren, die den Weg einer Berufslehre eingeschlagen und ohne grössere Verzögerungen abgeschlossen haben. Nach dem Ausschluss von Fällen mit fehlenden Werten in wichtigen Variablen sowie von Militär- oder Zivildienstleistenden (3.8\%) verbleiben 1'811 Lehrabgänger/innen im Sample, die Informationen über ihre Tätigkeit nach Lehrabschluss aufweisen.

Tabelle 1: Situation ein Jahr nach Lehrabschluss:

\begin{tabular}{|l|c|c|c|c|c|}
\hline & $\begin{array}{c}\text { Erwerbs- } \\
\text { tätig }\end{array}$ & $\begin{array}{c}\text { Weitere } \\
\text { Bildung }\end{array}$ & $\begin{array}{c}\text { Sprach- } \\
\text { aufenthalt }\end{array}$ & $\begin{array}{c}\text { Arbeits- } \\
\text { losigkeit }\end{array}$ & Total \\
\hline Abgänger duale Lehre $(\mathrm{N}=1487)$ & $74.5 \%$ & $15.7 \%$ & $3.3 \%$ & $6.5 \%$ & $100 \%$ \\
Abgänger Vollzeitberufsschule $(\mathrm{N}=324)$ & $36.4 \%$ & $46.0 \%$ & $6.8 \%$ & $10.8 \%$ & $100 \%$ \\
Alle (N=1'811) & $67.8 \%$ & $21.1 \%$ & $3.9 \%$ & $7.2 \%$ & $100 \%$ \\
\hline
\end{tabular}

Der Tabelle 1 zufolge sind rund ein Jahr nach Lehrabschluss zwei Drittel der Jugendlichen erwerbstätig, 21\% sind erneut in Ausbildung, 7\% sind arbeitslos und rund 4\% machen einen Sprachaufenthalt oder reisen. Es zeigen sich allerdings beträchtliche Unterschiede zwischen den Lehrabsolventen/innen dualer Berufslehren und denjenigen von Vollzeitberufsschulen.

Im Zentrum unseres Interesses steht der Berufswechselindikator, welcher für jede Person angibt, ob sie im erlernten Beruf arbeitet (Wert $=0$ ) oder nicht $(=1)$. Die Identifikation von Berufswechseln bedingt die Definition von Berufen und ist daher davon abhängig, wie eng der Begriff "Beruf» gefasst wird. Um eine möglichst objektive Definition von Berufswechseln zu erhalten, haben wir uns auf die Schweizerische Berufsnomenklatur SBN2000 gestützt und innerhalb dieser Nomenklatur jeweils den erlernten und den ausgeübten Beruf verglichen. ${ }^{8}$ Nur Wechsel auf der Zwei-Steller-Ebene, d.h. zwischen 36 Berufsgruppen, wurden als substanzielle Berufswechsel gewertet, was in einem Berufswechselanteil von $8.8 \%$ unter den 1'228 Erwerbstätigen resultiert. ${ }^{9}$ Tabelle 2 stellt die Berufswechselrate getrennt nach dual und vollzeitschulisch ausgebildeten Lehrabgänger/innen dar. Zu beachten ist, dass wegen fehlender Lohnangaben ${ }^{10}$ einige erwerbstätige Personen zusätzlich wegfallen, so dass für die Lohnregressionen 968 Fälle zur Verfügung stehen. 
Tabelle 2: Erwerbstätige und Berufswechsel

\begin{tabular}{|c|c|c|c|c|c|}
\hline & \multicolumn{3}{|c|}{ Abgänger duale Lehre } & \multirow[b]{2}{*}{$\begin{array}{c}\text { Abgänger } \\
\text { Vollzeit- } \\
\text { berufsschule }\end{array}$} & \multirow[b]{2}{*}{ Alle } \\
\hline & $\begin{array}{c}\text { Verbleib im } \\
\text { Lehrbetrieb } \\
(49.1 \%)\end{array}$ & $\begin{array}{c}\text { Neuer Betrieb } \\
(50.9 \%)\end{array}$ & $\begin{array}{c}\text { Insgesamt } \\
(100 \%)\end{array}$ & & \\
\hline Anteil Berufswechsel & $0 \%$ & $16 \%$ & $7.0 \%$ & $25.4 \%$ & $8.8 \%$ \\
\hline
\end{tabular}

Die Stichprobe der analysierten Lehrabgänger/innen weist folgende Merkmale auf: 53\% sind weiblich, $81 \%$ haben Eltern, die in der Schweiz geboren wurden, die Hälfte der Befragten mit ausländischen Eltern wurde selbst in der Schweiz geboren; insgesamt $82 \%$ haben eine duale Berufslehre absolviert (gegenüber $18 \%$ Vollzeitberufschulabsolventen), 57\% kommen aus der Deutschschweiz (gegenüber $32 \%$ bzw. $11 \%$ aus der französisch- und italienischsprachigen Schweiz) und 22\% (49\%) haben parallel zur dualen (vollzeitschulischen) Lehre die Berufsmaturität absolviert. Die Verteilung der erlernten Berufe ist nahe an derjenigen der Statistik der beruflichen Grundbildung 2006 (BFS, 2007). ${ }^{11}$ Am häufigsten ist die kaufmännische Lehre: unter den dualen Ausgebildeten haben $31 \%$ einen Abschluss als Kaufmann/Kauffrau, unter den Vollzeitschulabgänger/innen sind 67\% Handelsdiplomand/innen. ${ }^{12} \mathrm{Da}$ aufgrund der relativ tiefen Fallzahl in den multivariaten Schätzungen nicht detailliert für die einzelnen Berufe kontrolliert werden kann, wurden die Lehrberufe zusätzlich nach verschiedenen Eigenschaften klassiert. Zum einen wurden Lehrberufsgruppen auf der 1Steller-Ebene der Schweizerischen Berufsnomenklatur SBN2000 (mit und ohne Zusatzunterscheidung nach Lehrdauer) definiert. Zum anderen wurden dichotome Variablen gebildet, welche das intellektuelle Anforderungsniveau des Lehrberufs gemäss Stalder (2005) und die Höhe der mit der Ausbildung verbundenen Nettokosten für die Ausbildungsbetriebe (Mühlemann, Wolter, Fuhrer und Wüest, 2007; Schweri, Mühlemann, Pescio, Walther, Wolter \& Zürcher, 2003) abbilden. Als weitere Information wurde die berufsspezifische und sprachregionale Arbeitslosenrate des Lehrberufs (2-Steller-Ebene) aus der Schweizerischen Arbeitskräfteerhebung SAKE den einzelnen Personen zugespielt. Die Angaben $\mathrm{zu}$ den Lehr- und Erwerbsbetriebsgrössen stammen aus dem Betriebs- und Unternehmensregister des BFS und wurden dem TREE-Datensatz zugespielt. Zusätzlich zu den eingangs erwähnten soziodemografischen Charakteristika wie Geschlecht und Migrationsstatus werden weitere Informationen zum Hintergrund des Elternhauses aus der PISA-Befragung verwendet, nämlich ob die zuhause gesprochene Sprache der PISA-Testsprache entspricht oder nicht, der höchste erreichte Bildungsstand von Mutter oder Vater, und die Erwerbssituation der Eltern als Indikator für die Nähe zum Arbeitsmarkt. ${ }^{13}$ Als Indikatoren für die kognitiven Fähigkeiten der Befragten benutzen wir den in PISA durch Leistungstests ermittelten «Reading Literacy»-Score sowie die Lehrabschlussnote am Ende der Ausbildung. Eine erste deskriptive Auswertung des PISA-Testscores zeigt, dass Berufswechsler/innen und Arbeitslose gegenüber denjenigen mit 
einer Stelle auf dem Beruf, weiterer Ausbildung oder einem Sprachaufenthalt einen signifikant tieferen Wert aufweisen. Schliesslich bedienen wir uns individueller Einschätzungen zu Lehre und Erwerbssituation, die in den verschiedenen TREE-Wellen erhoben wurden: die allgemeine Zufriedenheit mit der eigenen Ausbildungssituation während der Lehre (Skala von 1 bis 7), wie sehr die Befragten die Tätigkeit in ihrem Beruf mögen, ebenfalls während der Lehre gefragt (Skala von 1 bis 4), sowie die Einschätzung der Nützlichkeit des in Lehrbetrieb und Berufsschule Erlernten in ihrer aktuellen Erwerbstätigkeit. Innerhalb der Gruppe der Berufswechsler geben knapp die Hälfte eine gute bis sehr gute Anwendbarkeit des in Berufsschule und Lehrbetrieb Erlernten an, etwas mehr als die Hälfte eine weniger gute bis schlechte Anwendbarkeit. Bei den auf dem Beruf arbeitenden Personen sind es dagegen rund 95\%, die angeben, das Erlernte gut bis sehr gut anwenden zu können.

\section{Schätzmethodik}

Wir prüfen die Relevanz möglicher Determinanten von Berufswechseln anhand eines multinomialen Entscheidungsmodells (multinomiales Logit ${ }^{14}$ ). Diese Methode wird verwendet, wenn die abhängige Variable eine diskrete und ungeordnete Skala aufweist und mehr als zwei Kategorien umfasst, was in Transitionsphasen wie der zweiten Schwelle typischerweise der Fall ist (vgl. zu einem ähnlichen Vorgehen für Deutschland Riphahn, 2002). Die abhängige Variable bildet fünf verschiedene Transitionsmöglichkeiten (im Folgenden als "Alternativen» bezeichnet) ab: «Stelle auf dem Beruf», "Berufswechsel», "Arbeitslos», "Weitere Bildung», "Sprachaufenthalt/Reisen».

Ermittelt werden soll, wie eine Veränderung der unabhängigen Variablen (z.B. erlernter Beruf oder Ausländerstatus) ceteris paribus (d.h. bei Kontrolle für den Vektor von Kovariaten ) die Wahrscheinlichkeit beeinflusst, dass die Alternative $\mathrm{j}$ eintrifft: $\mathrm{j}=0,1,2,3$, 4. Das Modell wird mit dem Maximum-Likelihood-Verfahren geschätzt (Wooldridge, 2002, S. 497-499). Aus den Schätzresultaten werden die Effekte einzelner Variablen auf die Wahrscheinlichkeiten der jeweiligen Wahlalternative berechnet und als sogenannte durchschnittliche Marginaleffekte ausgewiesen. ${ }^{15}$

Der Effekt von Berufswechseln auf den Lohn wird mit einer linearen multiplen Regression (Ordinary Least Squares OLS) geschätzt. Die abhängige Variable ist dabei, wie in der Arbeitsmarktökonomik üblich, der logarithmierte Lohn, so dass die geschätzten Koeffizienten der unabhängigen Variablen als prozentuale Lohnveränderungen interpretiert werden können. Neben einer Reihe von Kontrollvariablen enthält die Lohngleichung eine binäre Berufswechselvariable und eine binäre Betriebswechselvariable als unabhängige Variablen. Auf diese Weise können die Effekte von Betriebs- und Berufswechseln separat geschätzt werden, was der Unter- 
scheidung von betriebs- und berufsspezifischem Humankapital Rechnung trägt.

Grundsätzlich sind zumindest zwei potentielle Probleme mit der konsistenten Schätzung des Berufswechsel-Koeffizienten verbunden (vgl. für den analogen Fall von Betriebswechseln von Wachter und Bender, 2006). Erstens unterscheiden sich Personen hinsichtlich Fähigkeiten, Begabung und Leistungsvermögen, in der Literatur als «inherent ability» bezeichnet. Falls Personen mit bescheideneren Fähigkeiten auf dem Arbeitsmarkt schlechter entlöhnt werden und zugleich eher den Beruf wechseln, dann besteht die Gefahr, dass die Lohnkonsequenzen von Berufswechseln zu negativ geschätzt werden. Tiefere Löhne von Berufswechslern wären dann zumindest teilweise auf ihre geringeren Fähigkeiten zurückzuführen, und nicht kausal auf den Berufswechsel. Dank der reichhaltigen Informationen des TREE-Datensatzes und der Verknüpfung mit PISA2000 können wir diesem Problem begegnen, indem PISA-Testscores, Lehrabschlussnoten und sozioökonomischer Hintergrund als unabhängige Variablen in die Schätzungen aufgenommen werden und somit die Heterogenität der Befragten hinsichtlich ihrer Fähigkeiten berücksichtigt wird.

Zweitens finden viele Berufswechsel selektiv, nämlich nur im Falle einer Lohnverbesserung statt. Dadurch wird der erwartete negative Lohneffekt der Humankapitaleinbusse infolge eines Berufswechsels möglicherweise abgeschwächt oder vollständig überlagert. Dies kann unter bestimmten Annahmen durch die Verwendung von Instrumentalvariablenschätzern oder Selektionskorrekturen behoben werden. Im vorliegenden Artikel wird auf entsprechende Methoden verzichtet, da der Fokus auf der Beschreibung des Effektes erfolgter Berufswechsel und nicht auf dem reinen, hypothetischen Humankapitaleffekt liegt. ${ }^{16}$ Bei der Interpretation der Resultate muss allerdings berücksichtigt werden, dass sich die geschätzte Lohnwirkung von Berufswechseln nicht auf andere Personen, für die kein Berufswechsel beobachtet wurde, übertragen lässt.

\section{Ergebnisse: Einflussfaktoren für die Situation ein Jahr nach der Lehre}

Tabelle 3 zeigt die Resultate von multinomialen Logit-Analysen der Determinanten verschiedener Situationen nach der Lehre. Die abhängige Variable hat fünf Kategorien, im Folgenden als Alternativen bezeichnet: «Stelle auf dem Beruf», «Berufswechsel», "Arbeitslosigkeit», «Weitere Bildung», "Sprachaufenthalt/Reisen». 
Tabelle 3: Multinomiales Logit für die Situation ein Jahr nach der Lehre: Nur duale Ausgebildete

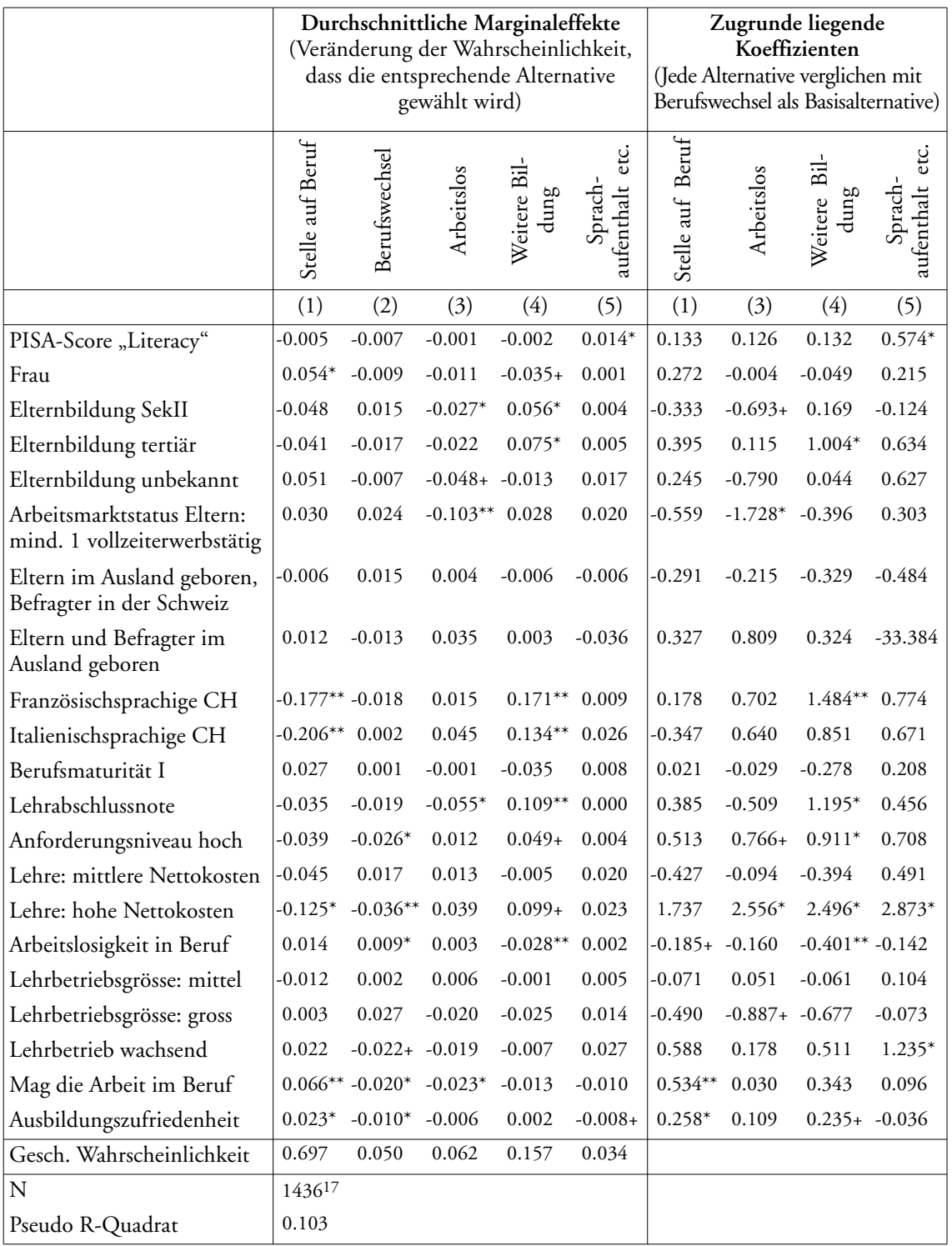

Bemerkung: ${ }^{* *} \mathrm{p}<0.01,{ }^{*} \mathrm{p}<0.05,+\mathrm{p}<0.1$

Die Referenzkategorien sind: Mann, obligatorische Schule als höchste Bildung der Eltern, kein Elternteil ist vollzeiterwerbstätig, Befragter und Eltern in der Schweiz geboren, deutsprachige Schweiz, keine Berufsmaturität, Anforderungsniveau unterhalb höchster Stufe, im Durchschnitt Nettoerträge der Ausbildung für Betriebe in gewähltem Beruf, Lehrbetriebsgrösse $<10$ Mitarbeiter. 
Die durchschnittlichen Marginaleffekte (linke Tabellenhälfte) repräsentieren die durchschnittlichen Veränderungen der geschätzten Wahrscheinlichkeit der jeweiligen Alternative bei Veränderung der entsprechenden unabhängigen Variable um eine Einheit. Die angegebenen Signifikanzen beziehen sich auf die Wahrscheinlichkeit einer Alternative gegenüber allen anderen Alternativen. Lesebeispiel: Frauen sind ceteris paribus um 5.4 Prozentpunkte wahrscheinlicher in der Kategorie "Stelle auf Beruf» anzutreffen als Männer. Die Summe der durchschnittlichen Marginaleffekte für alle fünf Alternativen summiert sich stets zu Null, da eine höhere Wahrscheinlichkeit für eine Alternative auf Kosten der anderen Alternativen geht. In der rechten Tabellenhälfte sind die Koeffizienten des zugrunde liegenden multinomialen Logit Modelles angegeben, welche zur Berechnung der Marginaleffekte verwendet wurden. Die Koeffizienten sind nur hinsichtlich Richtung und Signifikanzen in Bezug auf die Basisalternative "Berufswechsel» interpretierbar.

Bei den Determinanten von Berufswechseln dual ausgebildeter Lehrabgänger/innen kann aufgrund der durchschnittlichen Marginaleffekte (linke Tabellenhälfte, Alternative 2) als erstes festgestellt werden, dass personenspezifische Hintergrundsvariablen wie Migrationshintergrund, Geschlecht, elterliche Bildung etc. keinen signifikanten Einfluss ausüben. Dasselbe gilt für die Lehrabschlussnote und den PISA-Testscore, obwohl sich bei letzterem in der bivariaten Betrachtung noch signifikante Differenzen zwischen den Alternativen ergeben haben (vgl. "Daten und Operationalisierung»). Die Hypothese, dass es sich bei Berufswechsler/innen um eine Negativauswahl von weniger fähigen oder weniger produktiven Abgängern von dualen Lehren handelt, lässt sich demzufolge nicht bestätigen.

Es zeigt sich, dass nicht die kognitiven Fähigkeiten ${ }^{18}$, sondern vielmehr die Berufswahl (welche ihrerseits durch die kognitiven Fähigkeiten beeinflusst bzw. eingeschränkt sein mag) und somit verschiedene Charakteristika der durchlaufenen Ausbildung bedeutend sind für einen Berufswechselentscheid nach der Lehre. So wechseln Jugendliche aus Lehrberufen mit hohem intellektuellem Anforderungsniveau um 2,6 Prozentpunkte weniger wahrscheinlich den Beruf als Jugendliche aus schulisch weniger anspruchsvollen Lehrberufen. Eine ebenfalls kleinere Berufswechselneigung findet sich für Lehrabgänger/innen, deren Berufsausbildung für die ausbildenden Betriebe im Schnitt mit hohen Nettokosten während der Lehrzeit verbunden ist und wo somit keine Ausbildung über Bedarf stattfindet. Weiter findet sich, dass bei einer höheren sprachregional-berufsspezifischen Arbeitslosenquote eher ein Wechsel stattfindet. ${ }^{19}$ Die Lehrbetriebsgrösse hingegen zeigt keinen signifikanten Einfluss. Demnach sind Berufswechsel nicht gehäuft unter denjenigen Jugendlichen zu finden, welche evtl. aufgrund der kleinen Lehrbetriebsgrösse nach Lehrabschluss nicht weiterbeschäftigt werden konnten ${ }^{20}$. Als knapp signifikant zeigt sich jedoch das Betriebsgrössenwachstum des Lehrbetriebs in den vergangenen Jahren: Absolventen aus wachsenden Betrieben wechseln den Beruf nach Lehrabschluss seltener, was vermutlich darauf zurückzuführen ist, dass das Betriebsgrössenwachstum einen Indikator für allgemeine berufs- und branchenspezifische Beschäftigungsaussichten darstellt.

Nicht überraschend vermag die persönliche Bewertung der Lehre während der Ausbildung gewisse individuelle Berufswechseltendenzen aufzudecken: Ein 
Wechsel nach der Lehre ist wahrscheinlicher, je unzufriedener sich jemand während der Lehre zu seiner allgemeinen Ausbildungssituation äusserte und je weniger er/sie während der Lehre angab, die Arbeit in seinem Beruf zu mögen. Dies impliziert, dass Berufswechsel nicht nur durch äussere Faktoren forciert werden, sondern auch Ausdruck von veränderten Karriereplänen oder schlechtem Match mit dem Lehrberuf sein können und - in diesem Sinne - «freiwillig»stattfinden. 21

In Bezug auf die weiteren alternativen Situationen nach der Lehre ergeben sich folgende Resultate: Die Wahrscheinlichkeit, auf einer Stelle im erlernten Beruf $\mathrm{zu}$ arbeiten (Alternative 1), wird neben der Ausbildungszufriedenheit und der Freude an der Tätigkeit im Beruf während der Lehre auch von Faktoren bestimmt, welche für den oben diskutieren Berufswechselentscheid unbedeutend waren $^{22}$. So sind Frauen eher in einer Arbeitsstelle auf ihrem erlernten Beruf zu finden als Männer, sie verbleiben dafür eher weniger im Bildungssystem (Alternative 4). Die geringere Bildungsbeteilung der Frauen ist allerdings nur schwach signifikant. Weiter sind sprachregionale Unterschiede festzustellen: Jugendliche aus der frankophonen und italienischen Schweiz arbeiten mit geringerer Wahrscheinlichkeit auf dem Beruf und verbleiben eher im Bildungssystem. Gleiches gilt für Lehrabgänger/innen aus Berufen mit hohen Nettokosten der Ausbildung: sie wechseln nicht nur signifikant weniger den Beruf, sondern sind nach der Lehre im Vergleich zu Abgänger/innen rentabler Ausbildungsberufe generell mit tieferer Wahrscheinlichkeit auf dem Arbeitsmarkt anzutreffen; statt auf dem Beruf zu arbeiten, besuchen sie eher eine weitere Ausbildung. Separate Datenauswertungen zeigen, dass viele dieser Absolventen aus vorwiegend technischen Berufen (Maschinenindustrie, Informatik) direkt an eine höhere Fachschule oder Fachhochschule gehen. Die Bildungswahrscheinlichkeit wird zudem erhöht durch eine bessere Lehrabschlussnote. Auch das Bildungsniveau der Eltern ist ein einflussreicher Bestimmungsfaktor für eine weitere Bildungskarriere nach Lehrabschluss; je höher es ist, desto eher verbleiben die Jugendlichen im Bildungssystem.

Die Arbeitslosigkeitswahrscheinlichkeit (Alternative 3) wird neben einer schlechteren Lehrabschlussnote und geringerer Freude an der Tätigkeit im Beruf auch durch ein tieferes elterliches Bildungsniveau und, interessanterweise, durch eine geringe elterliche Arbeitsmarktpartizipation zum Zeitpunkt der PISA-Befragung signifikant erhöht. 23

In der rechten Tabellenhälfte sind die zugrunde liegenden Koeffizienten des multinomialen Logit Modells dargestellt. An den Koeffizienten sind Richtung und Signifikanz der unabhängigen Variablen für den paarweisen Vergleich mit der Basisalternative «Berufswechsel» herauszulesen. Aufschlussreich ist beispielsweise der direkte Vergleich zwischen der Berufswechsel- und Arbeitslosigkeitswahrscheinlichkeit. Lehrabgänger/innen aus Berufen mit hohem Anforderungsniveau und/oder hohen Nettokosten sind gemäss den Marginaleffekten in der linken Tabellenhälfte zwar nicht per se eher arbeitslos. Im direkten Vergleich der 
beiden Alternativen zeigt sich aber nun, dass sie signifikant eher als Abgänger/innen aus weniger anspruchsvollen oder kostenintensiven Berufen arbeitslos sind, anstatt den Beruf zu wechseln. Dies ist vermutlich so zu interpretieren, dass Absolvierende anspruchsvoller Berufe lieber auf eine Stelle im erlernten Beruf warten, als den Beruf zu wechseln, da dies mit einer erheblichen Lohneinbusse einhergehen dürfte und ein späterer Wechsel zurück in den erlernten Beruf schwierig sein könnte. Separate Auswertungen unter Einschluss des im erlernten Beruf zu erwartenden Lohnes deuten in eine ähnliche Richtung: Je tiefer der erwartete Lohn auf dem Lehrberuf, desto wahrscheinlicher ist ein Berufswechsel, je höher der erwartete Lohn, desto wahrscheinlicher wird Arbeitslosigkeit im Vergleich zum Berufswechsel. ${ }^{24}$

Tabelle 4: Multinomiales Logit für die Situation ein Jahr nach der Lehre (inklusive Abgänger-linnen aus Vollzeitberufsschulen)

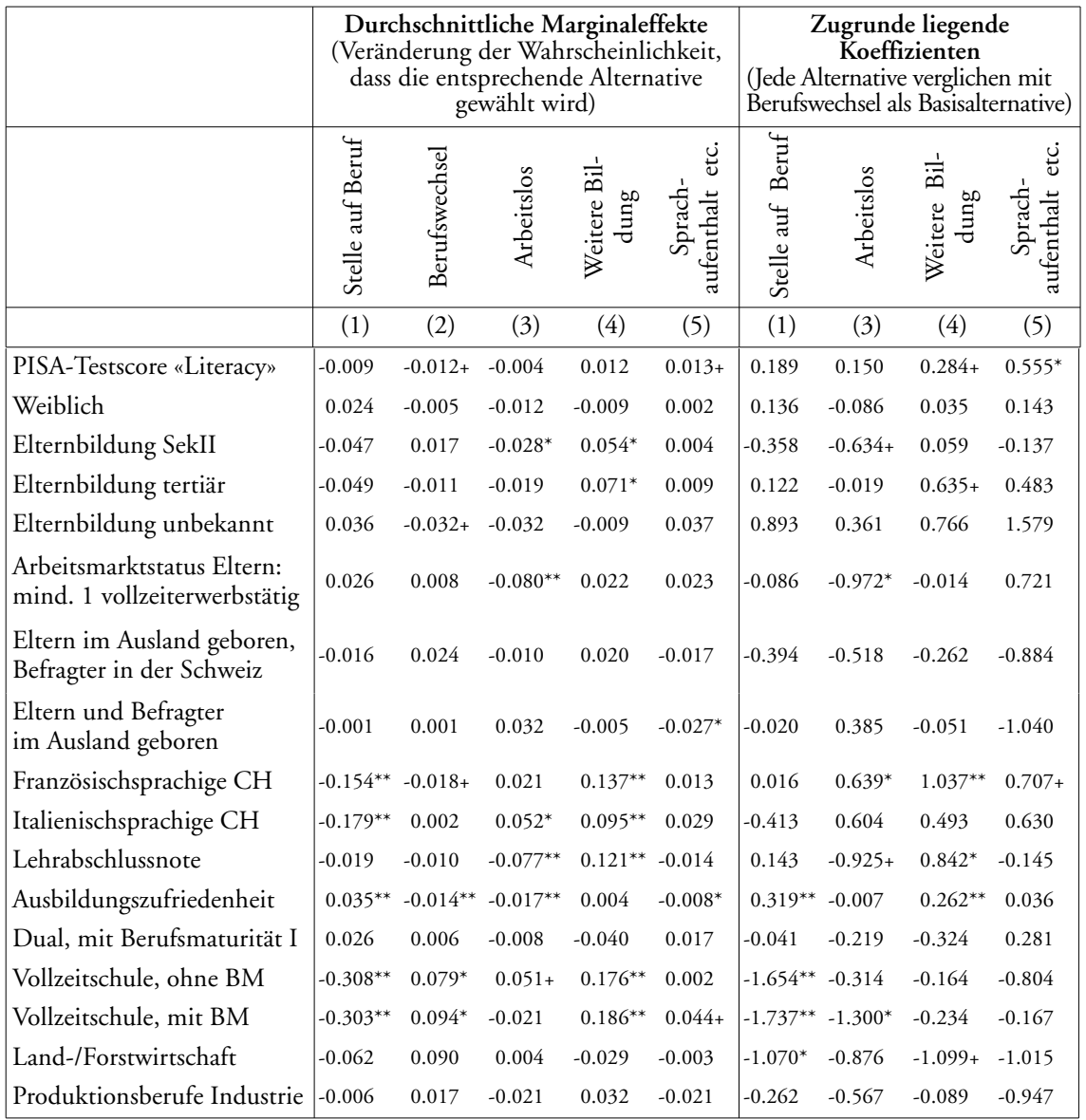




\begin{tabular}{|l|cccccc|cccc|}
\hline Technische Berufe, IT & $-0.108^{* *}-0.042^{* *}$ & 0.013 & $0.129^{* *}$ & 0.008 & $0.952+$ & $1.345^{*}$ & $1.741^{* *}$ & $1.391^{*}$ \\
Berufe des Baugewerbes & 0.078 & -0.000 & -0.021 & -0.029 & $-0.028^{*}$ & 0.168 & -0.302 & -0.167 & -1.116 \\
Handel-/Verkehrsberufe & $0.092^{*}$ & 0.002 & -0.026 & $-0.070^{*}$ & 0.002 & 0.173 & -0.421 & -0.472 & 0.033 \\
Gastgewerbe / persönl. DL & 0.024 & 0.058 & -0.010 & $-0.073^{*}$ & 0.000 & $-0.635+$ & -0.816 & $-1.162^{* *}$ & -0.686 \\
Gesundheitsberufe & $0.145^{* *}$ & $-0.039^{*}$ & -0.033 & $-0.062+$ & -0.012 & $1.331+$ & 0.488 & 0.641 & 0.690 \\
\hline Gesch. Wahrscheinlichkeit & 0.618 & 0.060 & 0.072 & 0.211 & 0.039 & & & & \\
\hline $\mathrm{N}$ & 1811 & & & & & & & & \\
Pseudo R-sq & 0.126 & & & & & & & & \\
\hline
\end{tabular}

Bemerkung: ${ }^{* *} \mathrm{p}<0.01,{ }^{*} \mathrm{p}<0.05,+\mathrm{p}<0.1$

Die Referenzkategorien sind: Mann, obligatorische Schule als höchste Bildung der Eltern, kein Elternteil ist vollzeiterwerbstätig, Befragter und Eltern in der Schweiz geboren, deutsprachige Schweiz, duale Lehre ohne Berufsmaturität, Beruf Kaufmann/-frau.

Die durchschnittlichen Marginaleffekte (linke Tabellenhälfte) repräsentieren die durchschnittlichen Veränderungen der geschätzten Wahrscheinlichkeit der jeweiligen Alternative bei Veränderung der entsprechenden unabhängigen Variable um eine Einheit. Die angegebenen Signifikanzen beziehen sich auf die Wahrscheinlichkeit einer Alternative gegenüber allen anderen Alternativen. In der rechten Tabellenhälfte sind die Koeffizienten des zugrunde liegenden multinomialen Logit Modelles angegeben, welche zur Berechnung der Marginaleffekte verwendet wurden. Die Koeffizienten sind nur hinsichtlich Richtung und Signifikanzen in Bezug auf die Basisalternative «Berufswechsel» interpretierbar.

Im Modell der Tabelle 4 wurde dieselbe Analyse unter Einschluss der Abgänger/innen von Vollzeitberufsschulen durchgeführt. Anstelle von Charakteristika der Berufslehre und des Lehrbetriebs wurde hier direkt für die Lehrberufsgruppe kontrolliert. Die Resultate zeigen, dass Vollzeitberufsschulabgänger/innen im Vergleich zu dual Ausgebildeten signifikant eher auf einer Stelle arbeiten, welche nicht ihrer Berufsausbildung entspricht (Alternative 2). Die Differenz beträgt 7,9 Prozentpunkte für Vollzeitschulabsolventen ohne Berufsmatur und 9,4 Prozentpunkte für solche mit Berufsmatur. Die Wahrscheinlichkeit, dass sie ein Jahr nach Abschluss in einer Stelle auf dem erlernten Beruf arbeiten (Alternative 1), ist über 30 Prozentpunkte geringer als bei dual Ausgebildeten, dafür ist die Wahrscheinlichkeit weiterer Bildung höher. Das Arbeitslosigkeitsrisiko ist nur für die Vollzeitschüler/innen ohne Berufsmatura erhöht, vermutlich weil sie keine oder kaum betriebliche Erfahrung aufweisen. ${ }^{25}$ In Bezug auf die schulischen bzw. kognitiven Fähigkeiten bleiben die meisten Ergebnisse aus Tabelle 3 bestehen: Personen mit besseren Lehrabschlussnoten verbleiben eher im Bildungssystem und sind weniger wahrscheinlich arbeitslos. Ebenfalls schwach signifikant ist bei Einschluss der Vollzeitschüler nun erstmals der Effekt des PISATestscores auf die Berufswechselwahrscheinlichkeit: je höher er ist, desto weniger wahrscheinlich wird der Beruf gewechselt. Beim direkten Vergleich der Wahlalternativen Arbeitslosigkeit und Berufswechsel (rechte Tabellenhälfte) zeigt sich ein (schwach) signifikanter Effekt in Richtung Berufswechsel statt Arbeitslosigkeit bei besseren Lehrabschlussnoten. In Bezug auf die Berufswechselwahrscheinlichkeit zeigen sich zudem Unterschiede zwischen den verschiedenen Berufsgruppen: Im Vergleich zur Referenzkategorie der Kaufmänner und -frauen nehmen Abgänger/innen aus technischen Berufen inkl. Informatik sowie aus 
den Gesundheitsberufen signifikant seltener einen Berufswechsel nach der Lehre vor. Es handelt sich dabei um Berufe, bei denen sich ein hoher Anteil an berufsspezifischem Humankapital vermuten lässt und bei denen eine grosse Arbeitskräftenachfrage herrscht.

\section{Die Lohnfolgen von Berufwechseln}

Dem Regressionsmodell (1) in Tabelle 5 zufolge haben duale Lehrabgänger/innen mit einem Berufswechsel im Schnitt einen 5,1\% tieferen Lohn als jene, die auf dem erlernten Beruf arbeiten, aber ebenfalls den Lehrbetrieb verlassen haben. Der Verbleib im Lehrberuf wird somit mit einem Lohnzuschlag für das berufsspezifische Humankapital «belohnt», wobei dieser Lohnvorteil aus vorne genannten Gründen eher unterschätzt sein dürfte. Hingegen zeigt sich ceteris paribus kein Lohnvorteil für den Verbleib im Lehrbetrieb.

Tabelle 5: Lohnregressionen (OLS) 26

\begin{tabular}{|c|c|c|c|c|}
\hline & (1) & (2) & (3) & $(4)$ \\
\hline \multicolumn{5}{|l|}{$\begin{array}{l}\text { Stelle (Referenzkategorie: Duale Lehre, arbeitet in neuem } \\
\text { Betrieb, aber auf dem erlernten Beruf) }\end{array}$} \\
\hline $\begin{array}{l}\text { Duale Lehre, neuer Betrieb und Berufswechsel } \\
\text { Interaktion Berufswechsel }\end{array}$ & $-0.051^{* *}$ & 0.005 & $-0.051^{* *}$ & $-0.047^{* *}$ \\
\hline x Gelerntes schlecht anwendbar & & $-0.095^{*}$ & & \\
\hline Duale Lehre, Stelle im Lehrbetrieb & 0.006 & 0.008 & 0.006 & 0.001 \\
\hline \multicolumn{5}{|l|}{ Interaktion Stelle im Lehrbetrieb } \\
\hline x Gelerntes schlecht anwendbar & & -0.033 & & \\
\hline Gelerntes schlecht anwendbar & & 0.027 & & \\
\hline Vollzeitberufsschule, Stelle auf Beruf, & & & $-0.033+$ & \\
\hline Vollzeitberufsschule, Berufswechsel & & & $-0.053+$ & \\
\hline Ungelernt (ohne Berufsbildung) & & & & $-0.089^{* *}$ \\
\hline Berufsmaturität I (dual) & -0.011 & -0.009 & -0.011 & \\
\hline Lehrabschlussnote & -0.008 & -0.015 & -0.006 & \\
\hline Lehrabschlussnote unbekannt & -0.009 & -0.013 & -0.007 & \\
\hline PISA-Testscore „Literacy“ & $0.018^{* *}$ & $0.018^{* *}$ & $0.014^{*}$ & $0.020^{* *}$ \\
\hline Elternbildung Sek II & -0.005 & -0.005 & -0.004 & 0.004 \\
\hline Elternbildung tertiär & 0.000 & -0.002 & -0.001 & 0.007 \\
\hline Elternbildung unbekannt & $0.049^{*}$ & $0.043+$ & $0.051^{*}$ & 0.036 \\
\hline Arbeitsmarktstatus Eltern: mind. 1 vollzeiterwerbstätig & 0.020 & 0.028 & 0.025 & 0.028 \\
\hline Weiblich & $-0.042^{* *}$ & $-0.045^{* *}$ & $-0.036^{* *}$ & $-0.043^{* *}$ \\
\hline \multicolumn{5}{|l|}{$\begin{array}{l}\text { Stelle (Referenzkategorie: Duale Lebre, arbeitet in neuem } \\
\text { Betrieb, aber auf dem erlernten Beruf) }\end{array}$} \\
\hline Eltern im Ausland geboren, Befragter in der Schweiz & 0.025 & 0.023 & 0.022 & 0.015 \\
\hline Eltern und Befragter im Ausland geboren & -0.009 & -0.010 & -0.005 & 0.010 \\
\hline Spricht zuhause nicht Testsprache & 0.024 & 0.024 & 0.030 & 0.022 \\
\hline Französischsprachige $\mathrm{CH}$ & -0.000 & -0.000 & -0.003 & 0.002 \\
\hline Italienischsprachige $\mathrm{CH}$ & $-0.096^{* *}$ & $-0.105^{* *}$ & $-0.107^{* *}$ & $-0.095^{* *}$ \\
\hline Erwerbsbetriebsgrösse: mittel & $0.023^{*}$ & $0.022^{*}$ & $0.024^{* *}$ & $0.020^{*}$ \\
\hline
\end{tabular}




\begin{tabular}{|llllc|}
\hline Erwerbsbetriebsgrösse: gross & $0.061^{* *}$ & $0.062^{* *}$ & $0.061^{* *}$ & $0.057^{* *}$ \\
Erwerbsbetriebsgrösse unbekannt & 0.033 & $0.065^{*}$ & 0.034 & $0.045^{*}$ \\
Erlernter Beruf (Referenzkategorie: Berufe des & & & & $\begin{array}{c}\text { Erwerbsberuf } \\
\text { (2Steller) }\end{array}$ \\
Managements und der Administration [3 Lehrjahre]) & & & & \\
$\quad$ & & & & \\
$\quad$ Land- und forstwirtschaftliche Berufe & $-0.102^{* *}$ & $-0.100^{* *}$ & $-0.103^{* *}$ & \\
$\quad$ Produktionsberufe der Industrie [3 Lehrjahre] & $-0.031+$ & $-0.041^{*}$ & $-0.033+$ & \\
$\quad$ Produktionsberufe der Industrie [4 Lehrjahre] & -0.009 & -0.015 & -0.015 & \\
Technische sowie Informatikberufe [4] & 0.007 & 0.003 & 0.007 & \\
$\quad$ Berufe des Bau- und Ausbaugewerbes [3] & 0.022 & 0.020 & 0.021 & \\
$\quad$ Berufe des Bau- und Ausbaugewerbes [4] & -0.013 & 0.010 & -0.011 & \\
$\quad$ Handels- und Verkehrsberufe [2] & $-0.152^{* *}$ & $-0.160^{* *}$ & $-0.158^{* *}$ & \\
$\quad$ Handels- und Verkehrsberufe [3] & $-0.045^{*}$ & $-0.045^{*}$ & $-0.049^{* *}$ & \\
\hline Berufe des Gastgewerbes und persönlicher Dienstleistungen [2] & $-0.096^{* *}$ & $-0.107^{* *}$ & $-0.099^{* *}$ & \\
Berufe des Gastgewerbes und persönlicher Dienstleistungen [3] & $-0.084^{* *}$ & $-0.083^{* *}$ & $-0.089^{* *}$ & \\
$\quad$ Berufe des Managements und der Administration [2] & -0.049 & -0.053 & $-0.073^{*}$ & \\
$\quad$ Gesundheitsberufe & -0.017 & -0.014 & -0.017 & \\
Konstante & $3.190^{* *}$ & $3.214^{* *}$ & $3.171^{* *}$ & $3.011^{* *}$ \\
\hline N & 885 & 801 & 968 & 964 \\
Korr. R-Quadrat & 0.258 & 0.265 & 0.239 & 0.278 \\
\hline
\end{tabular}

Bemerkungen: Abhängige Variable logarithmierter Bruttostundenlohn. Signifikanzniveaus ${ }^{* *} \mathrm{p}<0.01,{ }^{*}$ $\mathrm{p}<0.05,+\mathrm{p}<0.1$

Referenzkategorien übriger Variablen: keine Berufsmaturität, obligatorische Schule als höchste Bildung der Eltern, kein Elternteil ist vollzeiterwerbstätig, Befragter und Eltern in der Schweiz geboren, deutsprachige Schweiz, Erwerbsbetriebsgrösse $<10$ Mitarbeiter.

In Modell (2) wurde zusätzlich die individuelle Bewertung der Nützlichkeit des Erlernten für die aktuelle Stelle in Form einer binären Variable «gut bis sehr gut / weniger gut bis schlecht anwendbar» ins Modell aufgenommen und jeweils mit dem Berufswechselstatus interagiert. Das Resultat der Schätzung zeigt: gar keine Lohneinbusse erfahren Berufswechsler/innen, welche das Erlernte in ihrer aktuellen Stelle gut bis sehr gut anwenden können. Diejenigen aber, die in ihrer neuen Tätigkeit wenig anwenden können, verlieren 9,5\% gegenüber einer Stelle auf dem Beruf. Die auf der Grundlage der Berufsnomenklatur identifizierten Berufswechsel führen demnach nur in etwa der Hälfte der Fälle dazu, dass das berufsspezifische Humankapital nicht mehr eingesetzt werden kann, so dass eine erhebliche Lohneinbusse resultiert. Für die Nicht-Berufswechsler hat die Anwendbarkeit des Erlernten keinen signifikanten Einfluss auf den Lohn.

Einem separaten Modell 27 zufolge spielt bei Berufswechseln auch der Lehrberuf eine Rolle: Berufswechsler/innen aus landwirtschaftlichen Berufen, nichttechnischen Produktionsberufen, Handel, Gastgewerbe sowie persönlichen Dienstleistungsberufen scheinen gegenüber ihren jeweiligen Lehrberufskollegen/innen im Schnitt keine Lohneinbussen in Kauf nehmen zu müssen (beispielsweise verdient eine ausgelernte Coiffeuse im Verkauf nicht unbedingt weniger als eine Coiffeuse auf ihrem Beruf), wogegen Absolventen/innen aus kaufmännischen Lehren und dem Baugewerbe nach einem Berufswechsel 10\% bis $12 \%$ weniger verdienen als auf dem Beruf (unter den Abgängern aus techni- 
schen Berufen und der Informatik finden kaum Wechsel statt, daher ist keine Lohndifferenz schätzbar). Ein Lohnverlust von rund $10 \%$ der kaufmännisch Ausgebildeten bei einem Berufswechsel erscheint realistisch, wenn man die Lohndifferenzen zwischen den Berufen betrachtet (z.B. Modell 1): Wenn sie auf dem Beruf arbeiten, haben kaufmännische Angestellte gegenüber Verkäufern/innen mit einer zweijährigen Lehre im Schnitt gut 15\% höhere Löhne.

Ein interessanter Vergleich ergibt sich zwischen dualer und vollzeitschulischer Berufsbildung (Modell 3). In Tabelle 4 hat sich gezeigt, dass Absolventen/innen von Vollzeitberufsschulen insgesamt eine höhere Berufswechselwahrscheinlichkeit aufweisen. Die Hypothese, dass ihnen ihre Ausbildung eine höhere Mobilität auf dem Arbeitsmarkt ermöglicht, bestätigt sich in Modell (3) nicht eindeutig. Generell verdienen sie weniger als dual Ausgebildete mit demselben Lehrberuf $(-3,3 \%)$. Bei einem Berufswechsel weisen sie dafür vergleichsweise kleinere Lohneinbussen auf und verdienen nach einem Berufswechsel gleich viel wie duale Berufswechsler/innen mit demselben Ausbildungsberuf.

Modell (4) enthält im Unterschied zu den vorangegangen Schätzungen die unabhängige Variable Erwerbsberuf (statt des Lehrberufs). ${ }^{28}$ Dies erlaubt den direkten Vergleich der Löhne von Berufswechslern mit jenen von Ungelernten, welche dreieinhalb Jahre nach Abschluss der obligatorischen Schule keine zertifizierende Ausbildung abgeschlossen haben und arbeitstätig sind. Somit lässt sich in Modell (4) die Frage untersuchen, ob die absolvierte duale Lehre für die Berufswechsler in ihrem neuen Beruf zumindest teilweise lohnwirksam ist.

Zwar weisen Berufswechsler/innen 4,7\% tiefere Löhne auf als ihre Arbeitskollegen/innen, welche den ausgeübten Beruf in einer Berufslehre erlernt haben. Zugleich zeigt sich jedoch, dass die Löhne der Berufswechsler/innen im Schnitt $4,2 \%$ und statistisch signifikant höher sind als diejenigen der Ungelernten im selben ausgeübten Beruf mit maximal dreieinhalb Jahren Berufserfahrung29, aber sonst gleichen Charakteristika. Dieser Lohnvorteil mag eher gering erscheinen, doch ist es wahrscheinlich, dass die Berufswechsler mit Lehrabschluss in den folgenden Jahren und Jahrzehnten gegenüber den Ungelernten zusätzlich eine günstigere Lohnentwicklung aufweisen.

Was die weiteren Variablen betrifft, ergeben sich folgende Resultate: Ein höherer PISA-Testscore hat über die Modelle hinweg einen eigenständigen, signifikant positiven Lohneffekt: Ein um eine Standardabweichung höherer Testscore erhöht den Lohn um rund 1,5\% bis 2\%. Offenbar misst der PISA-Literacy-Testscore Fähigkeiten, welche die Produktivität beeinflussen und sich im Lohn niederschlagen. Weiter verdienen weibliche Fachkräfte direkt nach der Lehre im Schnitt 4\% weniger als ihre männlichen Kollegen. Jugendliche im Tessin haben gegenüber Deutschschweizern und Romands einen rund 10\% tieferen Lohn. Sonstige individuelle Hintergrundsvariablen wie der Migrationshintergrund zeigen sich nicht als lohnrelevant, ebensowenig die Lehrabschlussnote oder ein Be- 
rufsmaturitätsdiplom. Bedeutend ist aber die Betriebsgrösse des Erwerbsbetriebs - je grösser, desto höher die Löhne - und die Berufsgruppe sowie die Lehrdauer des erlernten Berufs. Gegenüber der Referenzgruppe der gelernten Kaufmänner/Kauffrauen verdienen beispielsweise Abgänger/innen aus Berufen der Landwirtschaft, Handel, Gastgewerbe und persönliche Dienstleistungen signifikant weniger; für zweijährige Lehren der Handels- und Verkehrsberufe (v.a. Verkäufer/-in) beträgt die Differenz 15,2\%.

\section{Zusammenfassung und Diskussion}

Berufswechsel werden hauptsächlich von der abgeschlossenen Berufslehre beeinflusst: eine höhere Arbeitslosenquote im Lehrberuf und tiefe Nettokosten der Ausbildung für die Betriebe - ein Indikator für eine hohe Anzahl von Lehrstellen bzw. -abgänger/innen - führen eher zu Berufswechsel. Gehört der Lehrberuf zu den Ausbildungen mit hohem intellektuellem Anforderungsniveau oder durchschnittlich hohen Nettokosten für die ausbildenden Betriebe - beides ein Indiz für hohen Humankapitalaufbau und vergleichsweise hohe zu erwartende Löhne auf dem Beruf - dann wird ein Berufswechsel eher vermieden; es wird in diesem Fall sogar eher eine zeitweilige Arbeitslosigkeit in Kauf genommen. Dieses Verhalten ist vermutlich rational, da eine Rückkehr in den erlernten Beruf durch einen Wechsel erschwert werden dürfte. Weiter zeigt sich, dass ceteris paribus eher jene Jugendlichen einen Berufswechsel vornehmen, welche schon während der Lehre mit ihrer Ausbildung unzufriedener waren oder im Vergleich $\mathrm{zu}$ anderen die Arbeit in ihrem Beruf weniger mochten, wo also die Passung zwischen Lehrberuf und dem Jugendlichen nicht ideal war.

Lehrabschlussnoten und persönliche Hintergrundscharakteristika wie Arbeitsmarktpartizipation und Bildungsnähe der Eltern haben bei gegebener Lehrberufswahl keinen Einfluss auf die Berufswechselwahrscheinlichkeit am zweiten Übergang, zeigen sich aber bezüglich des Arbeitslosigkeitsrisikos und der weiteren Bildungsaspiration als bedeutende Einflussfaktoren. Für Jugendliche mit Migrationshintergrund werden nach Lehrabschluss keine unterschiedlichen Übergangsmuster festgestellt.

Bezüglich Lohnfolgen kann festgestellt werden, dass die beobachteten Berufswechsler im Schnitt 5\% weniger verdienen als ihre Lehrberufskolleg/innen. Da nicht kleinere, sondern substanzielle Berufswechsel analysiert wurden, sind diese negativen Lohnfolgen keine Überraschung. Sie sprechen dafür, dass die Lehre auch dem Aufbau von berufsspezifischem Humankapital dient, welches der Arbeitsmarkt beim Verbleib im Lehrberuf entsprechend vergütet. Dies lässt sich als eine "Prämie» interpretieren, die durch die Spezialisierung in einem Beruf zustande kommt. Der Berufswechsel-Effekt ist allerdings nicht homogen: Für Berufswechsler/innen, die das erlernte Wissen in ihrer Stelle als nützlich erachten, ist kein Lohnabschlag zu beobachten. Anscheinend können sie in der 
neuen Tätigkeit trotz Berufswechsel gemäss Berufsnomenklatur an ihre Lehre anknüpfen. Weiter zeigen getrennte Betrachtungen nach Lehrberufsgruppen teilweise Lohneinbussen von über 10\%, also hohe Prämien für das erworbene berufsspezifische Wissen (z.B. Beruf Kaufmann/-frau); Wechsler/innen aus Berufen mit tieferen Löhnen verdienen hingegen nicht signifikant weniger. Der in den Lohnregressionen geschätzte mittlere Lohn der Berufswechsler/innen ist allerdings als obere Grenze zu interpretieren und nur für die beobachteten Berufswechsler gültig.

In Bezug auf die Unterscheidung der Lehrform - dual oder vollzeitschulisch - zeigt sich für letztere eine höhere Berufsmobilität mit weniger Lohneinbussen. Allerdings verdienen sie in einer Stelle auf dem Beruf weniger als ihre dual ausgebildeten Lehrberufskollegen und sind auch eher arbeitslos: im Schnitt scheinen sie damit - zumindest am zweiten Übergang - gegenüber den dual Ausgebildeten auf dem Arbeitsmarkt keine Vorteile zu besitzen. Ein genereller Ausbau von vollzeitschulischen beruflichen Grundbildungen drängt sich aus dieser Sicht bildungspolitisch nicht auf.

Weiter wird deutlich, dass die absolvierte Lehre für die Berufswechsler trotz Lohneinbusse ökonomisch gesehen nicht «umsonst» war: in ihrer aktuellen beruflichen Tätigkeit verdienen sie immer noch signifikant höhere Löhne als Ungelernte mit sonst gleichen Charakteristika. Absolventen/innen einer beruflichen Grundbildung können demzufolge auch nach einem Berufswechsel einen Teil des erworbenen Humankapitals verwerten, was den Jugendlichen in gewissem Masse erlaubt, günstige Beschäftigungsperspektiven in anderen Berufen zu nutzen. Zweifellos ist es aber eine wichtige Aufgabe des Staates, insbesondere bei jenen beruflichen Grundbildungen, deren Absolvierende häufiger den Beruf wechseln, darauf zu achten, dass während der Ausbildung genügend transferierbares Humankapital vermittelt wird. Dies ist im Interesse der Jugendlichen, denen sich dadurch bei ungünstigen Beschäftigungsperspektiven im erlernten Beruf mehr Alternativen bieten. Zugleich ist es auch volkswirtschaftlich gesehen wünschbar, wenn die Jugendlichen vorteilhafte Betriebs- und Berufswechselmöglichkeiten wahrnehmen können, weil dadurch die gesamtwirtschaftliche Effizienz gesteigert wird.

Verschiedene interessante Anschlussfragen können durch die vorliegende Arbeit nicht beantwortet werden. Zunächst stellt sich die Frage, wie sich Berufswechsel am zweiten Übergang in Bezug auf Lohnprofil und Beschäftigungsperspektiven in der langen Frist auswirken. Bedeutsam wäre auch, mehr zu wissen über die Gründe und Auswirkungen von Berufswechseln im weiteren Verlauf der beruflichen Karriere. Spielt etwa der erlernte Beruf einige Jahre nach Lehrabschluss noch eine Rolle hinsichtlich des Wechselverhaltens und des Lohns? Unterscheiden sich die Berufswechselmuster von Personen mit unterschiedlichen Ausbildungen, beispielsweise von Ungelernten, Personen mit beruflicher Grundbildung und Personen mit tertiärer Bildung? Weiter stellt sich die Frage, wie sich 
die zukünftige Situation derjenigen darstellt, welche sich ein Jahr nach Lehrabschluss noch nicht im Arbeitsmarkt befunden haben.

Schliesslich sind im vorliegenden Datensatz zu wenige Beobachtungen vorhanden, um detaillierte Auswertungen nach Lehrberufen und Erwerbsberufen machen zu können, insbesondere um die Vielfältigkeit der Berufswechselmuster (von welchem Beruf in welchen Beruf) zu analysieren. Zur Untersuchung dieser Frage fehlt in der Schweiz ein grosses Jugendpanel mit entsprechender Stichprobengrösse, das Informationen zu Ausbildung und Erwerbsituation enthält.

\section{Anmerkungen}

1 Diese Studie wurde finanziert vom Bundesamt für Berufsbildung und Technologie (BBT) über das Leading House für Bildungsökonomie: Betriebliche Entscheidungen und Bildungspolitik.

Für hilfreiche Kommentare bedanken wir uns bei zwei anonymen Gutachtern sowie beim Herausgeber dieses Heftes, Gianni Ghisla. Alle verbleibenden Fehler liegen in der Verantwortung der Autoren.

2 TREE (Transitionen von der Erstausbildung ins Erwerbsleben) wird seit 2008 ko-finanziert durch den Schweizerischen Nationalfonds SNF und die Universität Basel. Von 2000 bis 2007 lag die finanzielle und/oder organisatorische Verantwortung für TREE - neben dem SNF - bei einem Konsortium der Erziehungsdirektionen der Kantone Bern, Genf und Tessin, beim Bundesamt für Berufsbildung und Technologie (BBT) sowie beim Bundesamt für Statistik (BFS).

3 Der Berufswechselbegriff ist im Gegensatz zum Konzept der ausbildungsinadäquaten Beschäftigung wertneutral (für einen Überblick zu Ausbildungsinadäquanz vgl. Büchel und Weisshuhn, 1997).

4 Der Anteil vollzeitschulischer Ausbildungen an allen Berufsausbildungen liegt in der Schweiz mit ca. 12 bis 18 Prozent der Eintretenden (je nach Berechnungsweise) im Jahr 2005 vergleichsweise tief (BBT 2009, Schweri und Müller 2008); dabei liegt der Anteil vollzeitschulischer Berufsausbildungen in der lateinischen Schweiz höher als in der Deutschschweiz.

5 Für eine alternative Operationalisierung der Spezifität von Humankapital basierend auf dem spezifischen Qualifikationsmix, der in verschiedenen Berufen benötigt wird, siehe Lazear (2004), Gathman und Schönberg (2007) und Backes-Gellner, Geel und Mure (2009).

6 Diese Darstellung ist vereinfachend. Beispielsweise sind nicht nur die aktuellen Löhne der beiden Stellen relevant, sondern das abdiskontierte erwartete Lebenseinkommen; die zukünftigen Perspektiven (erwartetes Lohnwachstum etc.) können einen tiefen Einstiegslohn kompensieren. Die Modelle können zudem in einem umfassenden Sinne verstanden werden, bei dem die Gesamtheit der Arbeitsbedingungen relevant ist, von denen Lohn ein wichtiger Bestandteil ist.

7 Für eine Übersicht über das Projekt TREE, zum Datendesign und Stichprobe vgl. TREE (2008), für Projektergebnisse zum zweiten Übergang vgl. Bertschy, Böni und Meyer (2007).

8 Dabei wurden die Lehrberufe (kodiert nach dem Bildungsstatistischen Informationssystems BIS) mit Hilfe des Thesaurus der Schweizerischen Berufsnomenklatur (SBN) in die Berufs-Stammcodes der SBN überführt, sowie die Zuteilung von erlerntem und ausgeübtem Beruf innerhalb dieser Nomenklatur auf ihre Konsistenz hin überprüft und wenn nötig bereinigt. 
9 Bei einer Berufswechseldefinition auf 3-Steller-Ebene, also mit nächst höherem Detaillierungsgrad (88 verschiedene Berufsgruppen) wären es $9.5 \%$ statt $8.8 \%$ Berufswechsler/innen.

10 Fehlende Lohnangaben sind hauptsächlich auf die Befragungsprozedur zurückzuführen. Ein Teil der Fragen zur Erwerbstätigkeit (unter anderem die Lohninformationen) wurde in einem schriftlichen Zusatzfragebogen abgefragt, welcher nach Durchführung des Telefoninterviews den Befragten nach Hause geschickt wurde, aber nicht immer ausgefüllt wurde.

11 Die Top-25 Lehrberufe in TREE sind alle unter den Top-30 der EFZ-Abschlüsse im Jahr 2006 in der Schweiz.

12 Weitere häufig vorkommende Lehrberufe $(\mathrm{N}>15)$ sind in absteigender Reihenfolge Verkäufer/in, Detailhandelsangestellte/r, Koch/Köchin, Polymechaniker/in, Elektromonteur/in, Coiffeur/Coiffeuse, Büroangestellte/r, Pharma-Assistent/in, Automatiker/in, Gärtner/in, Bäcker-Konditor/in, Dentalassistent/in, Hochbauzeichner/in, Maurer/in, Konstrukteur/in, Bauzeichner/in, Automonteur/in, Florist/in, Elektroniker/in, Zimmermann/Zimmerin.

13 Dies ist eine 0/1-Variable, welche den Wert 1 annimmt, wenn mindestens ein Elternteil vollzeitbeschäftigt ist.

14 Eine wichtige Annahme in multinomialen Logit Modellen wird als independence of irrelevant alternatives (IIA) bezeichnet. Sie impliziert, dass sich für eine konsistente Schätzung der Parameter durch das Hinzufügen einer weiteren Alternative die relative Wahrscheinlichkeit zwischen zwei Alternativen nicht verändern darf. Diese Annahme kann mit dem Wald-Test von Hausman und McFadden (1984) überprüft werden. Die Nullhypothese, dass die IIAAnnahme zutrifft, konnte in den dargestellten Modellen nicht abgelehnt werden kann. Negative Testwerte für einige Alternativen können nach Long und Freese (2006, S. 245) als Evidenz interpretiert werden, dass die Nullhypothese nicht verworfen werden kann.

15 Der durchschnittliche Marginaleffekt einer unabhängigen Variable gibt an, wie sich die geschätzte Wahrscheinlichkeit der Wahl von Alternative j über alle Individuen verändert, wenn sich die unabhängige Variable um eine Einheit ändert. Für die Berechnung mit STATA vgl. Bartus (2005).

16 Müller und Schweri (2009) zeigen, dass sich der in OLS-Regressionen gefundene negative Lohneffekt von Berufswechseln verstärkt, wenn die Selektivität von Berufswechseln in der Schätzung berücksichtigt wird. Dies bestätigt die Hypothese, dass Berufswechsel eher vorgenommen werden, wenn sie einen Lohnvorteil versprechen. Daher stellen die Resultate zum Lohneffekt von Berufswechseln im vorliegenden Artikel eine untere Grenze (in absoluten Werten) des Lohneffektes dar, der bei einem Berufswechsel einer zufällig ausgewählten Person zu erwarten wäre.

17 Aufgrund von fehlenden Angaben in den unabhängigen Variablen stehen von den 1487 Absolventen/innen dualer Lehren gemäss Tabelle 1 nur 1'436 zur Verfügung.

18 Einschränkend ist festzuhalten, dass die PISA-Literacy-Testscores nur einen bestimmten Ausschnitt der kognitiven Fähigkeiten einer Person messen.

19 Die Signifikanzen bleiben bestehen, wenn das Modell mit cluster-robusten Standardfehlern geschätzt wird.

20 Dieses Ergebnis bleibt auch ohne Kontrolle aller anderen Faktoren wie bspw. der Höhe der Nettokosten bestehen. Das Ergebnis bleibt ebenfalls stabil, wenn man die Absolvent/innen mit einer Stelle auf dem Beruf zusätzlich danach aufteilt, ob sie im ehemaligen Lehrbetrieb angestellt sind oder nicht. In einem multinomialen Logit mit einer zusätzlichen Wahlvariante hat die Lehrbetriebsgrösse zwar einen signifikant positiven Einfluss auf den Verbleib im Lehrbetrieb, hingegen keinen Effekt auf die Berufswechselwahrscheinlichkeit. Berufswechsler/innen kommen nicht signifikant aus grösseren oder kleineren Lehrbetrieben als blosse Betriebswechsler oder als solche, welche in ihrem ehemaligen Lehrbetrieb angestellt sind. 
21 So hat beispielsweise rund die Hälfte der Berufswechsler/innen bereits während der Lehre angegeben, später eher nicht auf dem erlernten Beruf weiterarbeiten zu wollen

22 In einem multinomialen Wahlmodell lässt sich nicht automatisch darauf schliessen, dass Variablen, welche die Alternative «Berufswechsel» beeinflussen, in spiegelverkehrter Weise die Alternative "Stelle auf Beruf» beeinflussen. Die Gegenwahrscheinlichkeit zur Wahrscheinlichkeit von «Berufswechsel» ist die Summe der Wahrscheinlichkeiten der übrigen Alternativen. Vgl. auch das Lesebeispiel unter Tabelle 3.

23 Es sind verschiedene Erklärungen denkbar, wie der Arbeitsmarktstatus der Eltern das Arbeitslosigkeitsrisiko ihrer Kinder beeinflusst, bspw. aufgrund fehlender sozialer Netzwerke oder wegen des frühen Kontakts mit Arbeitslosigkeit in der Familie.

24 Dazu wurde zuerst ein Lohnmodell anhand derjenigen Personen geschätzt, welche auf dem erlernten Beruf geblieben sind. Es wurden dabei als Kontrollvariablen Lehrberuf, Sprachregion und Geschlecht benutzt. Anschliessend wurde anhand der geschätzten Parameter der erwartete Lohn auf dem Beruf auch für diejenigen Personen vorhergesagt, welche einen Berufswechsel vorgenommen haben. Der zu erwartende Lohn wurde zusammen mit den anderen Kontrollvariablen (ohne berufsbezogene Variablen) in die Schätzung der Tätigkeit ein Jahr nach der Lehre einbezogen. Die Resultate sind auf Anfrage bei den Autoren erhältlich.

25 Zur Erlangung der Berufsmatura müssen beispielsweise Schüler/innen der Handelsmittelschulen, welche die Mehrheit der Vollzeitberufsschüler/innen ausmachen, einen betrieblichen Praxisaufenthalt oder entsprechende Berufserfahrung von netto 39 Wochen vorweisen können.

26 Die Fallzahlen zwischen den Modellen ändern sich aufgrund von fehlenden Werten in der Variable «Anwendbarkeit des Gelernten» (Modell 2) und dem Einschluss von Vollzeitberufsschülern (Modell 3) sowie Ungelernten (Modell 4). Die Koeffizienten der verschiedenen Modelle können daher streng genommen nicht direkt miteinander verglichen werden.

27 Diese Resultate sind aus Platzgründen nicht in einer eigenen Tabelle dargestellt, aber auf Anfrage bei den Autoren erhältlich.

28 Die Erwerbsberufe sind erneut als 2-Steller der Schweizer Berufsnomenklatur (39 Berufsgruppen) definiert.

29 Für den Vergleich von Ungelernten mit Lehrabsolventen wurde der Lohn von Ungelernten drei Jahre nach PISA-2000, also aus der TREE-Erhebung 2004 verwendet.

\section{Literatuverzeichnis}

Acemoglu, D. \& Pischke, J.-S. (1998). Why do firms train? Theory and evidence. Quarterly Journal of Economic 113, 79-119.

Acemoglu, D. \& Pischke, J.-S. (1999). Beyond Becker: Training in imperfect labour markets. Economic Journal 109, F112-F142.

Backes-Gellner, U., Geel, R. \& Mure, J. (2009). Berufliche Bildung und Mobilität - Erklärungen mit Hilfe des Skill-Weights Approach. Empirische Pädagogik 23 (4), (in Druck).

Bartus, T. (2005). Estimation of marginal effects using margeff. Stata Journal 5 (3), 309-329.

Becker, G. S. (1962). Investment in human capital: A theoretical analysis. Journal of Political Economy 70 (5), 9-49.

Bertschy, K., Böni, E. \& Meyer, T. (2007). An der zweiten Schwelle: Junge Menschen im Übergang zwischen Ausbildung und Arbeitsmarkt. Ergebnisübersicht des Jugendlängsschnitts TREE, update 2007. Bern: TREE.

Bertschy, K., Cattaneo, M. A. \& Wolter, S. C. (2008). PISA and the transition into the labour market. LABOUR 23, 11-137.

Bonnal, L., Mendes, S. \& Sofer, C. (2002). School-to-work transition: Apprenticeship versus vocational school in France. International Journal of Manpower 23 (5), 425-442. 
Bradley, S. \& Nguyen, A. N. (2004). The school-to-work transition. In G. Johnes \& J. Johnes (Hrsg.), International handbook of education economics. Cheltenham: Edward Elgar.

Buchmann, M. (1996). Berufliche Aufstiegschancen und Abstiegsrisiken im Wandel. Neuchâtel: BFS.

Büchel, F. (2002). Successful apprenticeship-to-work transitions: On the long term change in significance of the german school-leaving certificate. International Journal of Manpower 23 (5), 394-410.

Büchel, F. \& Neubäumer, R. (2001). Ausbildungsinadäquate Beschäftigung als Folge branchenspezifischer Ausbildungsstrategien. Mitteilungen aus der Arbeitsmarkt- und Berufsforschung 34 (3), 269-285.

Büchel, F. \& Weisshuhn, G. (1997). Ausbildungsinadäquate Beschäftigung der Absolventen des Bildungssystems. Berichterstattung zu Struktur und Entwicklung unterwertiger Beschäftigung in West- und Ostdeutschland. Volkswirtschaftliche Schriften 471/II. Berlin: Duncker \& Humblot.

Bundesamt für Berufsbildung und Technologie (BBT). (2009). Zahlen und Fakten 2009. Bern: BBT.

Bundesamt für Statistik (BFS). (2002). Für das Leben gerüstet? Nationaler Bericht der Erhebung PISA 2000. Neuchâtel: BFS.

Bundesamt für Statistik (BFS). (2007). Statistik der beruflichen Grundbildung 2006. Neuchâtel: BFS.

Clark, D. \& Fahr, R. (2002). The promise of workplace training for non-college bound youth: Theory and evidence from german apprenticeship. CEP discussion papers 0518 , Centre for Economic Performance, LSE.

Dustmann, C. \& Meghir, C. (2005). Wages, experience and seniority. Review of Economic Studies 72, 77-108.

Dustmann, C. \& Schönberg, U. (2007). Apprenticeship training and commitment to training provision. Economics of education working paper series 0032, University of Zurich, Institute for Strategy and Business Economics (ISU).

Fitzenberger, B. \& Spitz, A. (2004). Die Anatomie des Berufswechsels: Eine empirische Bestandsaufnahme auf Basis der BIBB/IAB-Daten 1998/1999. ZEW Discussion Papers 05/2004.

Franz, W., Inkmann, J., Pohlmeier, W. \& Zimmermann, V. (2000). Young and out in Germany: On the youths chances of labor market entrance in Germany. In D. G. Blanchflower \& R. B. Freeman (Hrsg.), Youth unemployment and joblessness in advanced countries (pp. 381-426). Chicago, IL: University of Chicago Press.

Gathmann, C. \& Schönberg, U. (2007). How general is human capital? A task-based approach. IZA discussion paper no. 3067, Institute for the Study of Labor (IZA).

Hartog, J., Jonker, N. \& van Ophem, H. (2002). Dual track or academic route for auditors: Does it matter? IZA discussion paper no. 640, Institute for the Study of Labor (IZA).

Hausman, J. \& McFadden, D. (1984). Specification Tests for the Multinomial Logit Model. Econometrica 52(5), 1219-1240.

Jovanovic, B. (1979). Job matching and the theory of turnover. Journal of Political Economy 87 (5), 972-990.

Kambourov, G. \& Manovskii, I. (2008). Rising occupational and industry mobility in the United States: 1968-97. International Economic Review 49 (1), 41-79.

Korpi, T. \& Mertens, A. (2003). Training systems and labor mobility: A comparison between Germany and Sweden. Scandinavian Journal of Economics 105 (4), 597-617.

Lazear, E. P. (2004). Firm-specific human capital: A skill-weights approach. NBER working paper No. 9679 (revised version 2004).

Leemann, R. J. \& Keck, A. (2005). Der Übergang von der Ausbildung in den Beruf: Die Bedeutung von Qualifikation, Generation und Geschlecht. Neuchâtel: BFS.

Long, J. S. \& Freese, J. (2006). Regression models for categorical dependent variables using STATA (2nd ed). College Station, TX: Stata Press. 
Mortensen, D. (1986). Job search and labor market analysis. In O. Ashenfelter \& R. Layard (Eds.), Handbook of labor economics (vol. 2). North-Holland.

Mühlemann, S., Wolter, S. C., Fuhrer, M. \& Wüest, A. (2007). Lehrlingsausbildung - ökonomisch betrachtet. Ergebnisse der zweiten Kosten-Nutzen-Studie. Chur/Zürich: Rüegger.

Müller, B. \& Schweri, J. (2009). The returns to occupation-specific human capital-evidence from mobility after apprenticeship. Diskussionspapier präsentiert am Kongress der European Society of Population Economics (ESPE) 2009, Sevilla, und am Kongress der European Association of Labour Economists (EALE) 2009, Tallinn.

Neal, D. (1995). Industry-specific human capital: Evidence from displaced workers. Journal of Labor Economics, 13 (4), 653-677.

Neal, D. (1999). The complexity of job mobility among young men. Journal of Labor Economics, $17(2), 237-261$.

OECD. (2000). From initial education to working life - making transitions work. Paris: OECD.

OECD. (2002). PISA 2000 technical report. Paris: OECD.

Parent, D. (2000). Industry-specific capital and the wage profile: Evidence from the national longitudinal survey of youth and the panel study of income dynamics. Journal of Labor Economics, 18 (2), 306-323.

Plug, E. J. S. \& Groot, W. (1998). Vocational education versus apprenticeship: exemplified by the dutch situation. Working paper series WP1198, Université d'Orleans, Targeted Socio-Economic Research Schooling, Training and Transition (TSER SST).

Riphahn, R. T. (2002). Residential location and youth unemployment: The economic geography of school-to-work transitions. Journal of Population Economics, 15 (1), 115-135.

Rubinstein, Y. \& Weiss, Y. (2007). Post school earnings: search versus human capital. In E. A. Hanushek \& F. Welch (Hrsg.), Handbook of the economics of education (vol. 1). Elsevier B.V.

Ryan, P. (1998). Is Apprenticeship better? A review of the economic evidence. Journal of Vocational Education and Training 50 (2), 289-329.

Ryan, P. (2001). The school-to-work transition: A cross-national perspective. Journal of Economic Literature, 39 (1), 34-92.

Schweri, J., Mühlemann S., Pescio Y., Walther B., Wolter S. C. \& Zürcher, L. (2003). Kosten und Nutzen der Lehrlingsausbildung aus der Sicht Schweizer Betriebe. Chur/Zürich: Rüegger.

Schweri, J. \& Müller, B. (2008): Die Ausbildungsbereitschaft der Betriebe: Entwicklungen 1995 bis 2005. Neuchâtel: BFS.

Shaw, K. (1987). Occupational change, employer change, and the transferability of skills. Southern Economic Journal 53 (3), 702-719.

Sheldon, G. (2005). Der berufstrukturelle Wandel der Beschäftigung in der Schweiz 1970-2000: Ausmass, Ursachen und Folgen. Neuchâtel: BFS.

Smits, W. \& Zwick, T. (2004). Why do business service firms employ fewer apprentices? A comparison between Germany and the Netherlands. International Journal of Manpower 25 (1), 36-54.

Stalder, B. E. (2005). Das intellektuelle Anforderungsniveau von 105 Berufslehren. Bern: TREE.

Topel, R. H. \& Ward, M. P. (1992). Job mobility and the careers of young men. Quarterly Journal of Economics 107 (2), 439-479.

TREE. (Hrsg.). (2008). Projekt-Dokumentation 2000-2007. Bern/Basel: TREE.

Von Wachter, T. \& Bender, S. (2006). In the right place at the wrong time: The role of firms and luck in young workers' careers. American Economic Review 96 (5), 1679-1705.

Werwatz, A. (2002). Occupational mobility after apprenticeship - how effective is the german apprenticeship system? Applied Economics Quarterly (Konjunkturpolitik), 48 (3-4), 279-303.

Winkelmann, R. (1996). Employment prospects and skill acquisition of apprenticeship-trained workers in Germany. Industrial and Labor Relations Review 49 (4), 658-672. 
Winkelmann, R. (2006). Qualifikationsspezifische Beschäftigungsperspektiven und berufliche Flexibilität. In A. Frick \& A. Wirz (Hrsg.), Berufsbildungsökonomie: Stand und offene Fragen. Bern: h.e.p. Verlag.

Wolter, St. C. \& Schweri, J. (2004). Ökonomische Aspekte der Organisation einer Berufslehre. Zeitschrift für Berufs- und Wirtschaftspädagogik 100 (1), 13-25.

Wooldridge, J. M. (2002). Econometric analyses of cross section and panel data. Cambridge/London: MIT Press.

Schlagworte: Berufsbildung, Berufswechsel, Übergänge, Arbeitsmarkt, PISA

\section{Changement de profession au moment de la transition de l'apprentissage au travail}

\section{Résumé}

Faisant référence aux données panel TREE, cet article analyse l'incidence, les raisons et les effets des changements professionnels au moment de la transition de l'apprentissage au travail. Environ une année après l'obtention du certificat, 9\% des apprentis diplômés exercent une profession sensiblement différente de celle qui a été apprise. Les facteurs principaux influençant ce changement sont internes à la profession étudiée, par exemple le niveau d'exigences et le chômage dans la branche, ainsi que la satisfaction relative à la formation. Les variables liées à l'origine personnelle et aux capacités spécifiques des diplômés, telles qu'elles ont été mesurées dans les examens de sortie ou dans Pisa, ont par contre un effet limité.

Ceux qui changent de profession gagnent en moyenne $5 \%$ de moins que ceux qui continuent à travailler dans la profession apprise. Ce résultat montre que pendant la formation de base une spécialisation intervient qui est par la suite valorisée: ce qui a été acquis pendant l'apprentissage comme spécifique à la profession est rémunéré par le marché du travail. Toutefois les effets sur les salaires ne sont pas homogènes. En moyenne, ceux qui changent de profession gagnent tout de même plus que les personnes non qualifiées.

En comparant les personnes qui ont suivi un apprentissage dual avec les personnes ayant suivi des écoles professionnelles à plein temps, il apparaît que ces dernières gagnent en général légèrement moins et montrent une probabilité plus élevée à changer de profession. Après un changement de profession, les différences salariales entre les deux groupes ne sont plus significatives.

Mots clés: Formation professionnelle, changement de profession, transition, marché du travail, PISA 


\title{
Cambiamento di professione al momento della transizione tra apprendistato e lavoro
}

\section{Riassunto}

Fondandoci sui dati panel TREE, analizziamo l'incidenza, le ragioni e gli effetti dei cambiamenti professionali al momento della transizione tra apprendistato e lavoro. Un anno dopo il diploma, circa il 9\% degli apprendisti esercitano una professione notevolmente differente da quella imparata. I fattori principali che influenzano tale cambiamento hanno a che vedere con le caratteristiche della stesa professione imparata, ad esempio il livello di difficoltà e di esigenze, la disoccupazione del settore così come la soddisfazione con la propria formazione. Variabili legate al background personale e alle capacità specifiche dei diplomati, così come sono misurate negli esami in uscita e da Pisa, mostrano per contro un effetto limitato.

Coloro che cambiano professione guadagnano in media il $5 \%$ in meno di coloro che continuano a lavorare nella professione appresa. Questo risultato suggerisce che durante la formazione di base ci sia una specializzazione che viene premiata in seguito: il capitale umano specifico alla professione acquisito durante l'apprendistato viene remunerato dal mercato del lavoro. Tuttavia gli effetti salariali non sono omogenei. In media, coloro che cambiano professione guadagnano comunque significativamente meglio delle persone senza una educazione post-obbligatoria.

Confrontando persone che hanno seguito un apprendistato duale con persone che hanno seguito delle scuole professionali a tempo pieno, si nota come questi ultimi guadagnano leggermente meno, in generale, e mostrano una probabilità più alta di cambiare professione. Dopo un cambiamento di professione non vi è più una differenza salariale significativa fra i due gruppi.

Parole chiave: Formazione professionale, cambiamento di professione, transizione, mercato del lavoro, Pisa.

\section{Occupational change at the transition from apprenticeship to work}

\begin{abstract}
Based on the youth panel data set TREE, we analyse the incidence, reasons for and effects of occupational changes at the transition from apprenticeship to work. One year after graduation, about 9 percent of the apprenticeship graduates are working in a notedly different occupation than the one they have learned. The main factors influencing occupational change are features of the learned occupation such as the level of requirements and unemployment in the occupation, as well as the level of satisfaction with the training. Personal background va-
\end{abstract}


riables and graduates' abilities as measured by grades and PISA test scores show little effect.

Occupational changers earn 5 percent less, on average, than those who are working in the learned occupation. This result suggests that there is a specialisation going on in apprenticeship training; the occupation-specific human capital acquired during apprenticeship will be rewarded by the labour market when working in the learned occupation. The wage effects are, however, not homogenous.

On average, occupational changers earn significantly more than people without post-compulsory education. Comparing persons who followed a dual apprenticeship with persons who attended school-based VET tracks, the latter earn slightly less in general and exhibit a higher probability to change occupation. After an occupational change, there are no significant wage differences between the two groups anymore.

Key words: Apprenticeship training, vocational education and training (VET), occupation, transition, labour market, PISA 